typeset using JPSJ.sty $<$ ver.1.0b $>$

\title{
Transition from Haldane Phase to Spin Liquid and Incommensurate Correlation in Spin-1/2 Heisenberg Chains
}

\author{
Shinji Watanabe* and Hisatoshi Yokoyama \\ Department of Physics, Tohoku University, Aramaki Aoba, Aoba-ku, Sendai 980-8578
}

(Received September 29, 2018)

\begin{abstract}
The bulk properties and impurity effect in the one-dimensional $S=1 / 2$ Heisenberg model with dimerization and next-nearest-neighbor exchange are studied by the density matrix renormalization group and exact diagonalization methods. This model widely covers the $S=1$ Heisenberg model, two-leg ladders, bond-alternating chains and the $S=1 / 2$ Heisenberg model. Almost the whole parameter space belongs to the Haldane phase. In the non-dimerizing limit a transition to a kind of spin liquid takes place, characterized by the delocalized spin polarization. This transition causes the distinct impurity effects between a spin-Peierls compound $\mathrm{CuGeO}_{3}$ and the Haldane systems. Experimental aspects of a bond-alternating compound $\mathrm{CuNb}_{2} \mathrm{O}_{6}$ are also discussed. Furthermore, the incommensurate correlation arising in this model is extensively studied, and a phase diagram is constructed.
\end{abstract}

KEYWORDS: spin gap, Haldane phase, spin-Peierls transition, incommensurate correlation, impurity effect, twoleg ladder, $\mathrm{CuGeO}_{3}, \mathrm{CuNb}_{2} \mathrm{O}_{6}, \mathrm{DMRG}$, exact diagonalization

\section{$\S 1$. Introduction}

Recent progress of experimental studies has shed light on a variety of intriguing features in low-dimensional magnets. Above all, spin gaps found in such materials arouse broad interests with relation to the pseudo spin gap of the high- $T_{\mathrm{c}}$ superconductors. We list some classes of quasi one-dimensional (1D) spin-gap systems with typical compounds: The Haldane systems (NENP), bondalternating systems $\left(\mathrm{CuNb}_{2} \mathrm{O}_{6}\right)$, spin-Peierls systems $\left(\mathrm{CuGeO}_{3}\right)$, two-leg ladder systems $\left(\mathrm{SrCu}_{2} \mathrm{O}_{3}\right)$, and so forth. Although these compounds have similar spin-gap properties without any magnetic order in low temperatures, the behaviors toward the elemental substitution are entirely different. ${ }^{1,2)}$ A tiny amount of nonmagnetic or magnetic impurity (less than a few percent) breaks the spin gaps of $\mathrm{CuGeO}_{3}{ }^{3,4)}$ and $\mathrm{SrCu}_{2} \mathrm{O}_{3},{ }^{5,6)}$ and brings about antiferromagnetic (AF) long-range orders. In contrast, some Haldane systems ${ }^{1,7)}$ and $\mathrm{CuNb}_{2} \mathrm{O}_{6}{ }^{8)}$ keep their spin-gap behaviors even when substitutions as much as $20-40 \%$ are made, and no signs of magnetic orders are observed.

As the origin of this difference, multiple factors can be considered: [1] Difference in the nature of spin states within chains, [2] higher-dimensional (interchain) magnetic couplings $\left.,{ }^{9}, 10\right)$ [3] couplings with lattice degrees of freedom, ${ }^{11)}$ and [4] other factors, for example, disorder induced by the impurities, etc. Among these, we notice the factor [1], allowing for the following facts. Various experimental results for the above compounds are systematically and quantitatively understood within pure 1D spin models. ${ }^{8,12-15)}$ Furthermore, in spite of the similar interchain couplings (about 10\% of the intrachain coupling ${ }^{15,16)}$ ), $\mathrm{CuGeO}_{3}$ and $\mathrm{CuNb}_{2} \mathrm{O}_{6}$ exhibit distinct

\footnotetext{
*E-mail: watanabe@cmpt01.phys.tohoku.ac.jp
}

behaviors toward the impurity, as mentioned.

The main purpose of this paper is to investigate the cause of the above impurity effect on the basis of $1 \mathrm{D}$ chains. Using the density matrix renormalization group (DMRG), exact diagonalization (ED) methods and the perturbation theory, we study the properties within 1D precisely, thereby discuss the interchain coupling and the lattice effect. In order to argue in a unified viewpoint, we consider a model which continuously connects all the above materials in its parameter space: The 1D spin$1 / 2$ Heisenberg model with next-nearest-neighbor (NNN) coupling and bond alternation, ${ }^{17-19)}$

$$
\begin{array}{r}
\mathcal{H}=J\left[\sum_{i=1}^{L / 2}\left(\boldsymbol{S}_{2 i-1} \cdot \boldsymbol{S}_{2 i}+\beta \boldsymbol{S}_{2 i} \cdot \boldsymbol{S}_{2 i+1}\right)\right. \\
\left.+\alpha \sum_{i=1}^{L} \boldsymbol{S}_{i} \cdot \boldsymbol{S}_{i+2}\right],
\end{array}
$$

where $J>0$, and $\alpha$ and $\beta$ are dimensionless parameters controlling the coupling strength (see Fig. 1).

First, we study the bulk properties of the ground state and low-lying excited states, to grasp the global feature the Hamiltonian possesses in its parameter space. Next, we study the properties of finite systems, namely segments separated by the impurities, paying attention to the edge condition. In particular, the local spin polarization is important to consider magnetic long-range orders. The key point of the present impurity issue is that in the transition area from the Haldane phase to the spin liquid phase the local spin polarization highly depends on the system size.

Another fascinating aspect of this model is the incommensurate (IC) correlation. So far, except for the case without dimerization $(\beta=1)^{20-22)}$ few properties of the IC correlation have been elucidated. ${ }^{23)}$ To complete the 
phase diagram of the IC correlation in the $\alpha-\beta$ plane is another important purpose of this paper.

This paper is organized as follows. In $\S 2$ we briefly

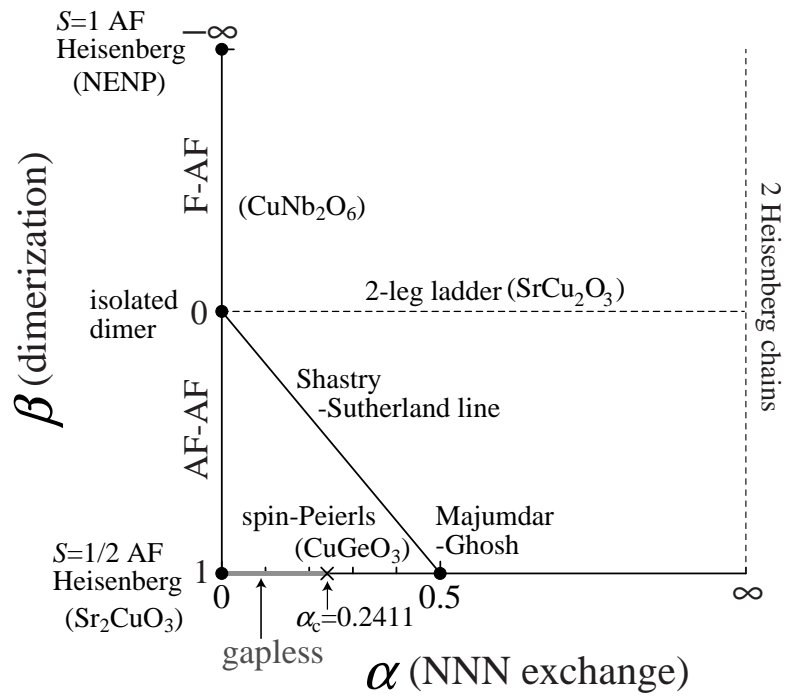

Fig. 1. Schematic diagram of the parameter space for the Hamiltonian eq. (1.1), spanned by two dimensionless parameters: $\alpha$ is of the next-nearest-neighbor exchange and $\beta$ of the bond alternation.

review the previous studies on the model eq. (1.1). In $\S 3$ the bulk properties of several quantities are studied widely in the $\alpha$ - $\beta$ plane. In $\S 4$ the incommensurate correlation is studied. In $\S 5$ we concentrate on the regime of spin-Peierls compounds $(\beta \sim 1)$, and consider the impurity effect in $\mathrm{CuGeO}_{3}$. In $\S 6$ the regime of Haldane phase is discussed with $\mathrm{CuNb}_{2} \mathrm{O}_{6}$ in mind. Finally, we summarize the main results in $\S 7$. In Appendix $A$, the relations between the two representations of the Hamiltonian, eq. (1.1) and eq. (2.3), are given. In Appendix B we supplement the explanation in $\S 3.1$ on the anomalous behaviors of the spin gap. In Appendix $\mathrm{C}$ we study the lowlying level structure for different boundary conditions, and show that the transition from the Haldane phase to the spin liquid occurs at $\beta=1$. In Appendix $\mathrm{D}$ we study the dynamical properties of the bond-alternating chains, in addition to various properties of monoclinic $\mathrm{CuNb}_{2} \mathrm{O}_{6}$.

\section{$\S 2 . \quad$ Model and Method}

The Hamiltonian eq. (1.1) is represented as a two-leg ladder with zigzag rungs as shown in Fig. 2. This model includes various classes of 1D spin systems as described below. The parameter of bond alternation $\beta$ is restricted to the range $-\infty \leq \beta \leq 1$, by taking account of the translational symmetry. For the open boundary condition (OBC), however, it is quite important whether the first and last bonds have the value $J$ or $\beta J$. This point will be discussed later. As for the parameter of the NNN exchange $\alpha$, we restrict the range to $0 \leq \alpha \leq \infty$ in this paper.

There are some special regions in the $\alpha-\beta$ plane as listed below (See also Fig. 1):

[1] $(\alpha, \beta)=(0,1)$ : The $S=1 / 2$ AF Heisenberg model.

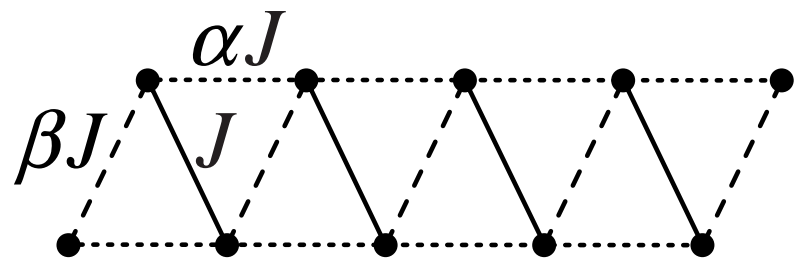

Fig. 2. Connectivity of the Hamiltonian eq. (1.1).

[2] $\beta=-\infty$ : The $S=1$ AF Heisenberg model.

[3] $\beta=2 \alpha(0 \leq \alpha \leq 0.5)$ : The so-called ShastrySutherland (SS) line. ${ }^{24)}$ In particular, the two ends $(0,0)$ and $(0.5,1)$ are (a) the isolated dimer point, (b) the Majumdar-Ghosh (MG) point, ${ }^{25)}$ respectively.

[4] $\beta=0$ : The two-leg ladder. The isotropic ladder $\left(J_{\text {leg }} / J_{\text {rung }}=\alpha=1\right)$ is represented by $(1,0)$.

[5] $\alpha=\infty$ : The model is decoupled to two $S=1 / 2 \mathrm{AF}$ Heisenberg chains with the doubled unit cell.

We start with the relation to the Haldane issue. On the $\beta$ axis $(\alpha=0)$, the Hamiltonian represents the AFferromagnetic $(\mathrm{F})$ bond-alternating chains for $\beta<0$. In the limit of $\beta \rightarrow-\infty$ (as the Hund coupling), $\mathcal{H}$ is exactly reduced to the $S=1$ Heisenberg model with a numeric factor $1 / 4$. In this viewpoint Hida studied the properties for finite values of $\beta(\alpha=0)$, using ED. $\left.{ }^{26}\right)$ He found that the properties of the Haldane phase continue even to the $\mathrm{AF}-\mathrm{AF}$ alternating region $(\beta>0)$. Later, by extending the non-local unitary transformation used for the $S=1$ systems, ${ }^{27)}$ Kohmoto and Tasaki ${ }^{28}$ ) and Takada ${ }^{29)}$ showed exactly that the hidden $Z_{2} \times Z_{2}$ symmetry is completely broken for the case [3(a)], and approximately that the Haldane phase continues to the limit [1].30)

On the other hand, there are many researches in view of the analogy between the Haldane systems and ladders. For example, White showed that the isotropic ladder point [4] belongs to the same phase with the Haldane system, using DMRG. ${ }^{31)}$ Recently using a variational matrix product method, Brehmer et al. studied the ground-state properties ${ }^{17}$ ) and low-energy excitations $^{18)}$ of eq. (1.1), and concluded that their trial function smoothly connects the AKLT model ${ }^{32)}$ and the MG model $[3(\mathrm{~b})]$. Thus, a wide range of the $\alpha-\beta$ space seems covered by the Haldane phase. In this phase the following conditions have to be satisfied:

- A unique singlet ground state with a finite singlettriplet excitation gap (Haldane gap) for the systems of $L=\infty$ or for the periodic boundary condition (PBC).

- Fourfold (pseudo-)degeneracy appears under the Haldane gap for the finite open chains due to the two localized $S=1 / 2$ spins at both edges. ${ }^{33)}$

- A hidden topological (string) long-range order (LRO) exists. ${ }^{34)}$

At the MG point $[3(\mathrm{~b})]$, the ground state for the $\mathrm{PBC}$ spontaneously breaks the translational symmetry and 
has twofold degeneracy:

$$
\begin{aligned}
& \psi_{1}=[1,2][3,4] \cdots[L-1, L], \\
& \psi_{2}=[2,3][4,5] \cdots[L, 1],
\end{aligned}
$$

where $[i, j]=\alpha(i) \beta(j)-\beta(i) \alpha(j)$. On the other hand, on the SS line $\beta=2 \alpha(0 \leq \alpha<0.5), \psi_{1}$ whose singlet pairs sit on the bonds with the larger exchange $J$ becomes the unique ground state, and $\psi_{2}$ is no longer an eigenstate.

On the $\alpha$ axis $(\beta=1),{ }^{20)}$ the excitation is gapless for $0 \leq \alpha \leq \alpha_{\mathrm{c}}$. Note that the gapless regime in the $\alpha-\beta$ plane is limited to this segment and $\alpha=\infty$. The critical point $\alpha_{\mathrm{c}}=0.241167^{35)}$ is connected to the HaldaneShastry model, ${ }^{36}$ ) in the sense that the conformal invariance is realized. For $\alpha>\alpha_{\mathrm{c}}$, the ground state is twofold degenerate for $L \rightarrow \infty$, in accordance with the Lieb-Shultz-Mattis theorem. ${ }^{37)}$

The IC correlation arises in the $\beta<2 \alpha$ side of the SS line. ${ }^{20-23)}$ However, details including the boundary position have been still obscure. As far as the $\alpha$ axis $(\beta=1)$ is concerned, it is known that an IC wave number which maximizes $S(q)^{38)}$ appears for $0.52063 \leq \alpha \leq \infty .^{21,22)}$

Here, we comment on another and more popular representation in the spin-Peierls regime $(\beta \lesssim 1)$ :

$$
\mathcal{H}=\tilde{J} \sum_{i}\left\{\left[1-(-1)^{i} \delta\right] \boldsymbol{S}_{i} \cdot \boldsymbol{S}_{i+1}+\tilde{\alpha} \boldsymbol{S}_{i} \cdot \boldsymbol{S}_{i+2}\right\} .
$$

The Hamiltonians eqs. (1.1) and (2.3) are identical except that the space of eq. (2.3) is limited to $1 \geq \beta \geq-1$ in the $\alpha-\beta$ plane. We use each representation in its suitable situation. The relations between the two representations are summarized in Appendix A.

Lastly, we describe numerical methods used. The DMRG method for zero temperature ${ }^{39)}$ is a powerful method to calculate the ground-state and the low-energy properties accurately for low-dimensional systems. In this paper we adopt the finite size algorithm with OBC. The number of states kept for each block is up to $m=200$. The system size is usually $L \sim 200$, and is increased up to 400 according to necessity. The typical truncation error is $O\left(10^{-12}\right)$ for the region away from the $\alpha$ axis $(\beta=1)$. But it becomes $O\left(10^{-8}\right)$ as $\beta$ approaches 1 , where the correlation length diverges, and also in the incommensurate area. We show error bars when accuracy is required.

The ED (Lanczos and Householder) methods are used for supplementary calculations to DMRG, and to study dynamical and thermodynamic properties with a recursion procedure for the former. We check system-size dependence and make extrapolations from the data up to $L=26$ for the former properties and up to $L=18$ for the latter.

\section{§3. Global Features of the $\alpha-\beta$ Plane}

In this section, we study the bulk properties of several quantities widely in the $\alpha-\beta$ space. In $\S 3.1$ we consider the spin gap. The anomaly in the spin gap is a sign of the incommensurate correlation treated in $\S 4$. In $\S 3.2$ two-point correlation function is discussed. In $\S 3.3$ the order parameters of dimerization and of hidden topological (string) order are studied. We refer to the phase transition from the Haldane phase to a spin liquid at $\beta=1$.

\subsection{Spin gap}

Before discussing the results, we need to remark on the edge effect in the DMRG calculations of the gap. As is known, an open $S=1$ chain has free $S=1 / 2$ spins near both chain ends in the Haldane phase. These spins yield pseudo fourfold degenerate states (one singlet and three triplets) beneath the bulk Haldane gap. Therefore, in estimating the bulk gap by DMRG ${ }^{40)}$ extra $S=1 / 2$ spin had to be attached to both edges artificially to cancel the free spins. In the present case we take advantage of a similar but natural cancellation, by imposing the condition on the system that the edge bonds should be $J$ (the stronger one). For the bulk quantities, we use this boundary condition. We will discuss the effect of edge bond in Appendix C.2.

Figure 3 shows the $\beta$ dependence of the spin gap $\Delta$ for some values of $\alpha$ obtained by DMRG. The spin gap is obtained by $\Delta(L)=E(1 ; L)-E(0 ; L)$, where $E\left(S^{z} ; L\right)$ is the lowest energy with total $S^{z}$ and system size $L$. We extrapolate $\Delta(L)$ to the thermodynamic limit by using the polynomial fit:

$$
\Delta(L)=\Delta(\infty)+\sum_{n=1} \frac{c_{n}}{L^{n}}
$$

with $n \leq 8$ for $L=12,16,24,32,48,64,100,200,400$.

For $\alpha=0$, the result is consistent with those previously estimated by ED. ${ }^{26)}$ For $\beta \rightarrow-\infty$ the gap is smoothly reduced to $\Delta_{\mathrm{H}} / 4 J$, where $\Delta_{\mathrm{H}}=0.41050(2)^{40)}$ is so-called Haldane gap in unit of the exchange coupling for the $S=1$ AF Heisenberg chain.

At the isolated dimer point the gap becomes the maxi-

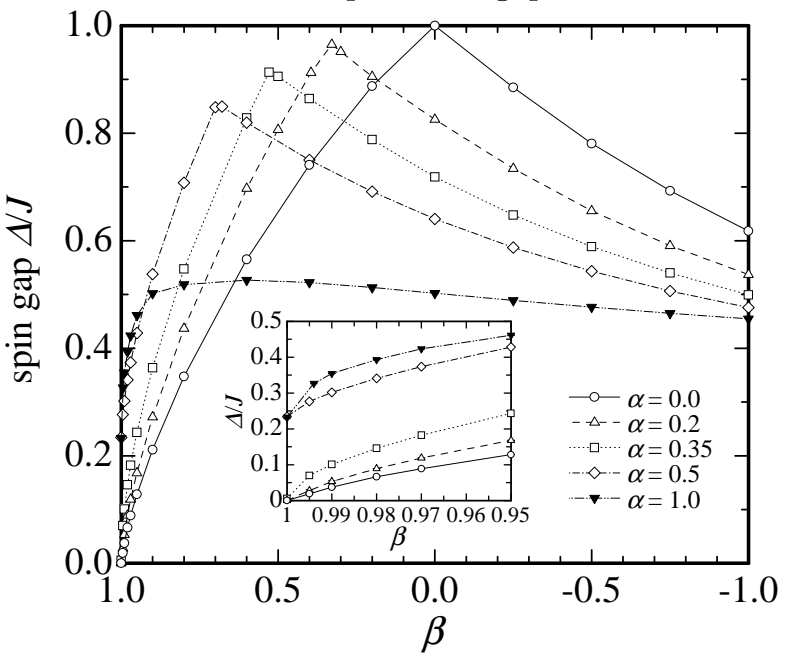

Fig. 3. $\beta$-dependence of the spin gap for some values of $\alpha$ estimated by the DMRG method. The values are obtained by the extrapolation of the finite systems of $L=12-400$. The inset shows the enlargement for $0.95 \leq \beta \leq 1$.

mum $J$ in the $\alpha-\beta$ plane. There, the model is reduced to the two-spin problem, so that the energy cost to break up one singlet into triplet is $J$. At this point $\Delta$ forms 
a cusp as seen in Fig. 3. In the following, we study this anomaly in connection with the incommensurate correlation treated in $\S 4$. For finite but small values of $\alpha$ and $\beta$, this anomaly can be traced by the second-order perturbation theory. The energy per bond for the ground state (singlet) and the lowest triplet branches are obtained respectively as,

$$
\begin{aligned}
\frac{E_{\mathrm{s}}}{J}= & -\frac{3}{4}-\frac{3}{16}(\beta-2 \alpha)^{2} . \\
\frac{E_{\mathrm{t}}(q)}{J}= & \frac{1}{4}-\frac{1}{4}\left(\beta^{2}+4 \alpha^{2}\right) \\
& -\left[\frac{1}{2}(\beta-2 \alpha)+\frac{1}{4}\left(\beta^{2}-4 \alpha^{2}\right)\right] \cos (2 q),
\end{aligned}
$$

where $q=n \pi / N(-N / 2<n \leq N / 2 ; n=$ integer $)$ and $N=L / 2$ (the number of dimers) with the lattice constant being unity. Note that for $\beta=2 \alpha$ cancellation occurs between $\alpha$ and $\beta$. For the ground state, $E_{\mathrm{s}}$ leads to the constant exact value of the SS line. ${ }^{24)}$ Meanwhile, for $E_{\mathrm{t}}(q)$ the hopping matrix element in eq. (3.2) vanishes, so that the singlet-triplet excitation forms a flat blanch. When a parameter $(\alpha$ or $\beta)$ is varied and crosses this line, the wave number minimizing $E_{\mathrm{t}}(q)$ switches from $q=\pi / 2$ to $0(\pi)$ discontinuously, due to the hopping term. Thus, the above dispersionless point corresponds to the cusp of the spin gap. On the other hand, the second term in eq. (3.2) survives even for $\beta=2 \alpha$; the spin gap decreases from $J$ even along the cusp line.

When $(\alpha, \beta)$ goes away from $(0,0)$, the cusp still remains as seen in Fig. 3. In the $\alpha-\beta$ plane, the cusp point deviates to the side of $\beta<2 \alpha$ with increasing $\alpha$, as shown in Fig. 12. This singular point is related to the incommensurate correlation, and will be taken up again in $\S 4$. We have confirmed by ED that the properties found by the above perturbation theory are preserved for $\alpha \lesssim 0.5$. On the other hand, for $\alpha \gtrsim 0.5$ with increasing $\beta$, the bottom of the triplet blanches does not switch directly from $\pi / 2$ to $0(\pi)$, but IC values of $q$ intervene. Thus, the cusp disappears. Actually, for $\alpha=1$ the gap changes smoothly from the Haldane limit $(\beta=-\infty)$ to $\beta \sim 1$ through the isotropic ladder point $(\beta=0)$, as depicted in Fig. 3. The supplement of this issue is given in Appendix B.

The inset of Fig. 3 shows the enlargement for $0.95 \leq$ $\beta \leq 1$. The $\delta$-dependence ${ }^{41)}$ of the gap has been discussed in relation to the spin-Peierls transition. ${ }^{13,42)}$ The gap drops steeply near $\beta=1$ even for the spontaneously gapped regime $\left(\alpha>\alpha_{\mathrm{c}}\right)$, as seen for $\alpha=0.35$ and 0.5 .

\subsection{Spin-spin correlation}

In this subsection, we discuss the spin-spin correlation function $S_{i j}=\left\langle S_{i}^{z} S_{j}^{z}\right\rangle$ in the ground state. Using the system of $L=280$ typically, we calculate $S_{i j}$ in the central part of the system. The 40 sites near the open edges are not used to eliminate edge effects.

In the gapped regime, where $S_{i j}$ decays exponentially for $\left.|i-j| \rightarrow \infty,{ }^{43}\right)$ we assume a simple form:

$$
\left\langle S_{i}^{z} S_{i+r}^{z}\right\rangle \propto \exp \left(-\frac{\xi}{r}\right) .
$$

Incidentally, we do not adopt the 2D Ornstein-Zernike type used for the $S=1$ chain, ${ }^{44}$ ) because it is not certain whether it is adequate also in the present model. The above fit successfully works for most cases except for $\beta \sim$ 1, where the algebraic decay is essential. In Fig. 4 typical cases of the exponential decay are shown: An AF-AF and a F-AF bond-alternating systems and the isotropic ladder, etc. The inset shows that the ranges of ill fit by eq. (3.3) are limited to several lattice spacings. The correlation length $\xi$ is obtained from this fit.

In Figs. 5(a) and 5(b) we plot $\xi$ for $-1 \geq \beta>-\infty$ and

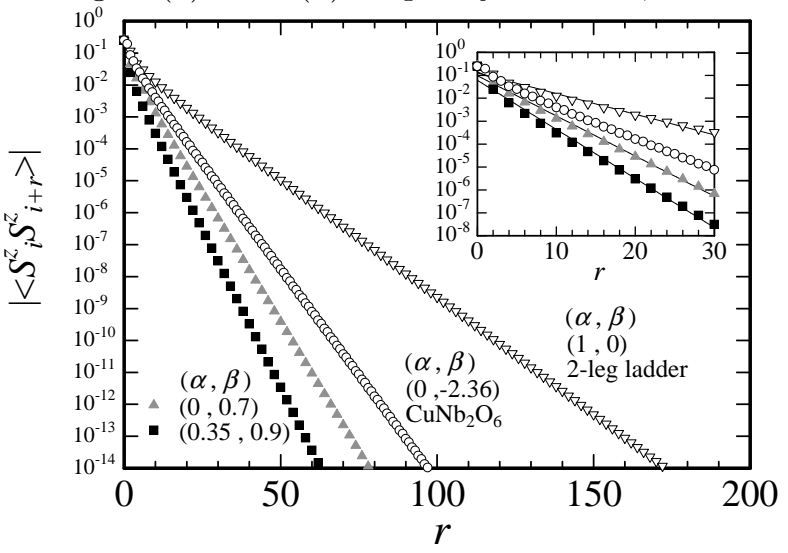

Fig. 4. Spin-spin correlation function versus $r$ for some typical cases of exponential decay. Since the fitted line for odd $r$ shifts a little from that for even $r$ for $\beta>0$, only the data for even $r$ are shown except for $(0,-2.36)$. The inset is the magnification of the small- $r$ regime with fitted lines.

$1 \geq \beta \geq-1$, respectively. In the Haldane limit $(\beta \rightarrow$ $-\infty), \xi$ is reduced to $2 \xi_{\mathrm{H}}$ [cross symbol in Fig. 5(a)], where $\xi_{\mathrm{H}}=6.03(1)^{40)}$ is the correlation length of the $S=1$ AF Heisenberg chain. The factor 2 arises due to the doubled unit cell. As $\alpha$ increases, $\xi$ approaches the value in the Haldane limit faster. This is because $\alpha$ does not work as frustration for $\beta<0$, but plays a cooperative role to stabilize the $\uparrow \uparrow \downarrow \downarrow$ structure.

As shown in Fig. 5(b) $\xi$ vanishes on the SS line $(\beta=2 \alpha)$, where $S_{i j}$ vanishes for $|i-j| \geq 2 .^{25)}$ Comparing with Fig. 3, we find the minimum point of $\xi$ does not coincide with the maximum point of the spin gap, except for the isolated dimer point. In the upper side of the SS line $(\beta<2 \alpha)$, the region exists where $\left|S_{i j}\right|$ weakly oscillates with an IC period. ${ }^{23)}$ We indicate the other commensurate-IC boundary - so far unknown - by arrows in Fig. 5(b). In this regime, the fit by eq. (3.3) becomes less accurate with increasing $\beta$ because of the frequent oscillation; possible errors thereof are shown by bars. We will discuss the IC correlation in $\S 4$.

Now, let us consider the vicinity of the $\alpha$ axis. In Fig. 6 we show $S_{i j}$ for $\beta=1$. For the gapless regime $S_{i j}$ shows algebraic decay (with some logarithmic correction $^{45)}$ ), as seen for $\alpha=0$ and 0.2. An important fact is that even in the gapped regime $\left(\alpha>\alpha_{\mathrm{c}}\right), S_{i j}$ shows a similar non-exponential decay for some distance. Of course, in a sufficiently long distance $S_{i j}$ decays exponentially. Therefore, we try to divide $S_{i j}$ into two parts. 

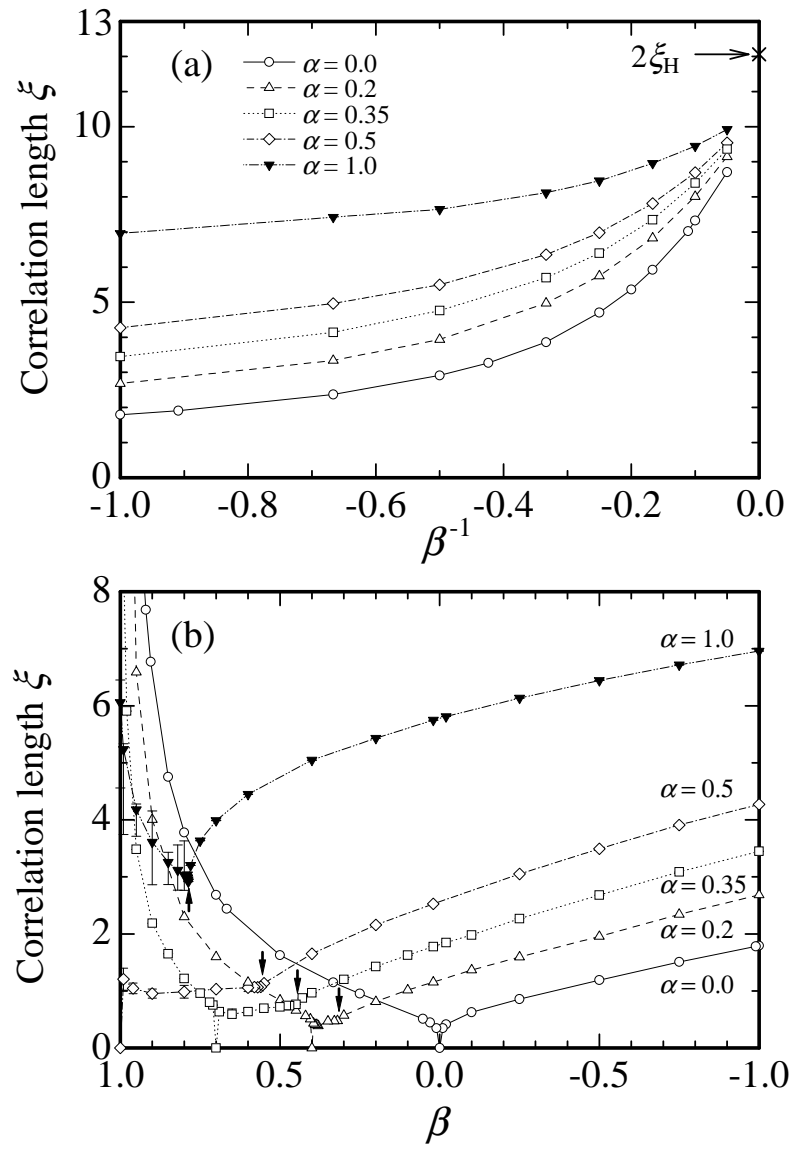

Fig. 5. Correlation length for some values of $\alpha$ as a function of (a) $1 / \beta$ for $-\infty<\beta \leq-1$, and of (b) $\beta$ for $-1 \leq \beta<1$. In (a) cross symbol at $\beta^{-1}=0$ indicates twice the correlation length of the $S=1 \mathrm{AF}$ Heisenberg model. ${ }^{40)}$ In (b) arrows stand for the commensurate-incommensurate boundary observed in $S_{i j}$. $\xi$ vanishes on the Shastry-Sutherland line $(\beta=2 \alpha)$.

The arrows in Fig. 6 show the minimal $r(\equiv \eta)$ which satisfies the condition that the error in the exponential fit for $r>\eta$ exceeds $10^{-3}$ (this value is for convenience). Thereby, we roughly estimate the boundary of the decaying behavior. For $\alpha<\alpha_{\mathrm{c}}, \eta=\infty$; even for $\alpha>\alpha_{\mathrm{c}}, \eta$ is still considerably large, e.g. $\eta=163$ for $\alpha=0.35$. In the vicinity of the MG point $(\xi=0) \eta$ decreases abruptly.

This tendency still remains in the vicinity of the $\alpha$ axis $(\beta \sim 1)$. There, the short-range correlation is controlled by the semi-algebraic decay, and poorly fitted by eq. (3.3). Therefore, the values of $\xi$ plotted in Fig. 5(b) have been estimated from $S_{i j}$ 's with sufficient large $|i-j|$. Such enhancement of the short-range spin correlation near the $\alpha$ axis is closely connected to the impurity effect in spin-Peierls systems. This will be discussed in $\S 5$.

\subsection{Order parameters}

We begin with an order parameter for dimerization defined by

$$
O_{\mathrm{D}}=\left|\left\langle\boldsymbol{S}_{2 i-1} \cdot \boldsymbol{S}_{2 i}\right\rangle-\left\langle\boldsymbol{S}_{2 i} \cdot \boldsymbol{S}_{2 i+1}\right\rangle\right| \cdot
$$

Figure 7 shows $O_{\mathrm{D}}$ versus $\beta$ for some values of $\alpha$.

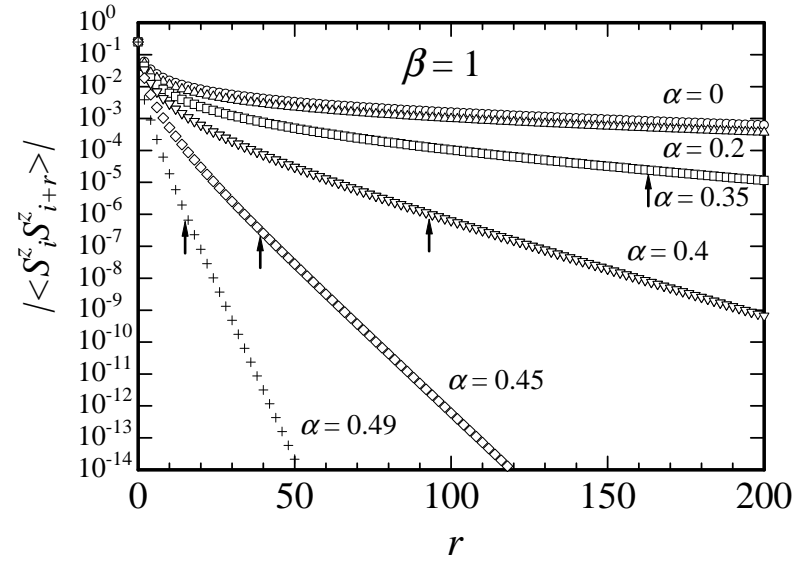

Fig. 6. Spin-spin correlation function $\left\langle S_{i}^{z} S_{i+r}^{z}\right\rangle$ as a function of $r$ for some values of $\alpha . \beta=1$. The arrow indicates $\eta$, which roughly divides $S_{i j}$ into two parts, of exponential decay $(r>\eta)$ and of semi-algebraic decay $(r<\eta)$. The data for even $r$ are shown for clarity.

First, we look at the cases for $\alpha=0$. As $\beta$ decreases from 1 , the dimerization abruptly becomes larger and continues increasing until $\beta=-1$, where $O_{\mathrm{D}}$ has the maximum value 0.8245165 . Since $O_{\mathrm{D}}=3 / 4$ for the isolated dimer point, moderate magnitude of ferromagnetic exchange between singlets further promotes the dimerization. ${ }^{22)}$ Beyond $\beta=-1 O_{\mathrm{D}}$ monotonically decreases and tend to the value $O_{\mathrm{D}}=0.600371010$ estimated for the $S=1$ AF Heisenberg chain, for $\beta \rightarrow-\infty$.

Next, for $0<\alpha<\alpha_{\mathrm{c}}$ there remain the absence of

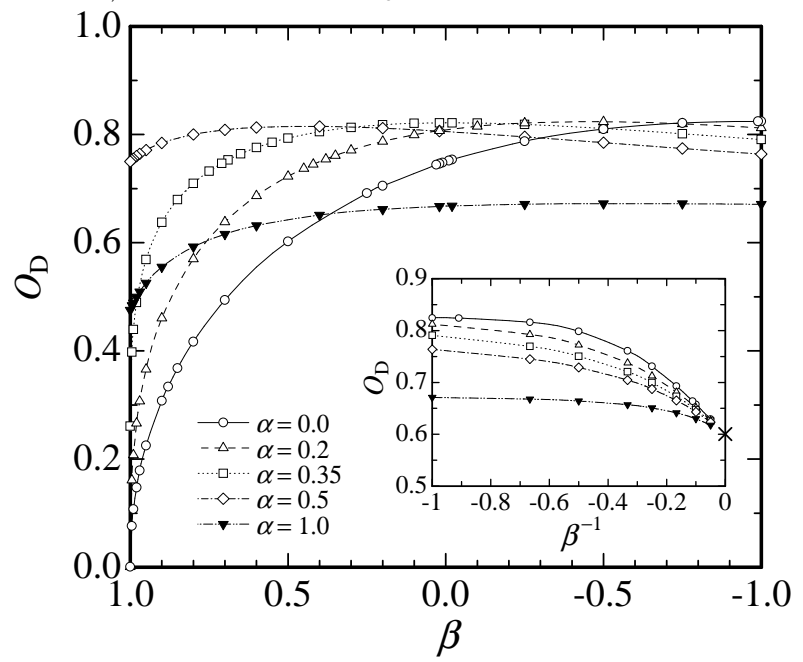

Fig. 7. Dimer order parameter as a function of $\beta(1 \geq \beta \geq-1)$ for some values of $\alpha$. Inset shows the same quantity for $\beta \leq$ -1 as a function of inverse $\beta$. The cross at $\beta^{-1}=0$ indicates the value for the $S=1$ Heisenberg chain: $\left(1-E_{\mathrm{H}}\right) / 4$, where $E_{\mathrm{H}}=-1.40148440390 .^{40)}$ On the SS line $(\beta=2 \alpha) O_{\mathrm{D}}=3 / 4$. Extrapolation is carried out from the DMRG data of $L=12-400$.

dimerization for $\beta=1$ and the abrupt increase for $\beta \sim 1$. On the other hand, for $\alpha>\alpha_{\mathrm{c}}, O_{\mathrm{D}}$ shows a finite value even for $\beta=1$ due to the spontaneous dimerization. 
With increasing $\alpha$ the maximum point of $O_{\mathrm{D}}$ shifts in the positive direction of $\beta$, although the maximum value is almost unchanged. This aspect is understood by the cooperative roles of $\alpha$ and $-\beta$, as mentioned in $\S 3.2$. In contrast to the case of $\beta=1,{ }^{22}$ ) there is no direct correspondence between the spin gap and $O_{\mathrm{D}}$. In Fig. 3 the spin gap shows the cusp, while $O_{\mathrm{D}}$ does not show any singular behavior, and smoothly connects the Haldane limit $(\beta=-\infty)$ and the limit of $\beta \rightarrow 1$.

Now, we turn to the string order parameter $O_{\mathrm{S}}$. The string correlation function to measure the topological LRO in the Haldane phase for $S=1$ systems $^{34)}$ was extended to the $S=1 / 2$ bond-alternating chain as, ${ }^{30)}$

$$
\begin{gathered}
O_{\mathrm{S}}(i-j)= \\
-\left\langle\left(S_{2 i-1}^{z}+S_{2 i}^{z}\right) \prod_{\ell=2 i+1}^{2 j-2} \exp \left(i \pi S_{\ell}^{z}\right)\left(S_{2 j-1}^{z}+S_{2 j}^{z}\right)\right\rangle .
\end{gathered}
$$

In this expression, ferromagnetic (weaker AF) bonds are assumed between the sites $2 i-1$ and $2 i$ and between ones $2 j-1$ and $2 j$. Among others, this definition is the most natural extension to the present system.

In Fig. 8 shown is $O_{\mathrm{S}}=\lim _{r \rightarrow \infty} O_{\mathrm{S}}(r)$ estimated by DMRG. As has pointed out for the $S=1$ case, ${ }^{40)}$ it is technically important to measure $O_{\mathrm{S}}(r)$ at a sufficient distance from the open edge, not to mention large $|r|$. Actually, for the cases of $\beta \sim 1$ and small $\alpha$, the required distance exceeds our chain length $(L=400)$. This technical difficulty prevents us from concluding decisively that $O_{\mathrm{S}}$ vanishes as $\beta \rightarrow 1$ for $\alpha<\alpha_{\mathrm{c}}$. We believe it occurs, however, taking account of the singular behaviors near $\beta=1$. On the other hand, for $\alpha>\alpha_{\mathrm{c}}, O_{\mathrm{S}}$ has a finite value; for the MG point, $O_{\mathrm{S}}=1 / 4$.

When $\beta$ decreases from $1, O_{\mathrm{S}}$ increases at first, has a maximum at a finite value of $\beta$, and then tends to the value for the $S=1$ case. Here again, the value for a larger $\alpha$ approaches faster to the Haldane limit. For $\alpha=1, O_{\mathrm{S}}$ varies gradually through the isotropic ladder point $(\beta=0)$. Like $O_{\mathrm{D}}$, the string order changes continuously keeping finite values from $\beta=-\infty$ to $\beta \sim 1$. This strongly suggests that the properties of the Haldane phase are kept in almost the whole $\alpha-\beta$ plane except just on the $\alpha$ axis.

In fact, similar conclusions have been already reached in the case of $\alpha=0$ by the ED and variational calculations, ${ }^{26,30)}$ and for $\alpha \neq 0$ by variation methods. ${ }^{17,19)} \mathrm{We}$ demonstrate that the transition from the Haldane phase to a spin liquid phase occurs at $\beta=1$ in the case of the SS line in Appendix. This discussion can be generalized to all the $\alpha-\beta$ plane. As we will see later, this "spin liquid phase" is characterized by the delocalized spin polarization, in contrast to the localized moment at the edges in the Haldane phase.

\section{§4. Incommensurate Correlation}

In this section we study the incommensurate (IC) correlation the model eq. (1.1) presents. In $\S 4.1$ and $\S 4.2$ we discuss the IC correlation with respect to $S_{i j}$ and $S(q)$, respectively. In $\S 4.3$ we construct a comprehensive phase

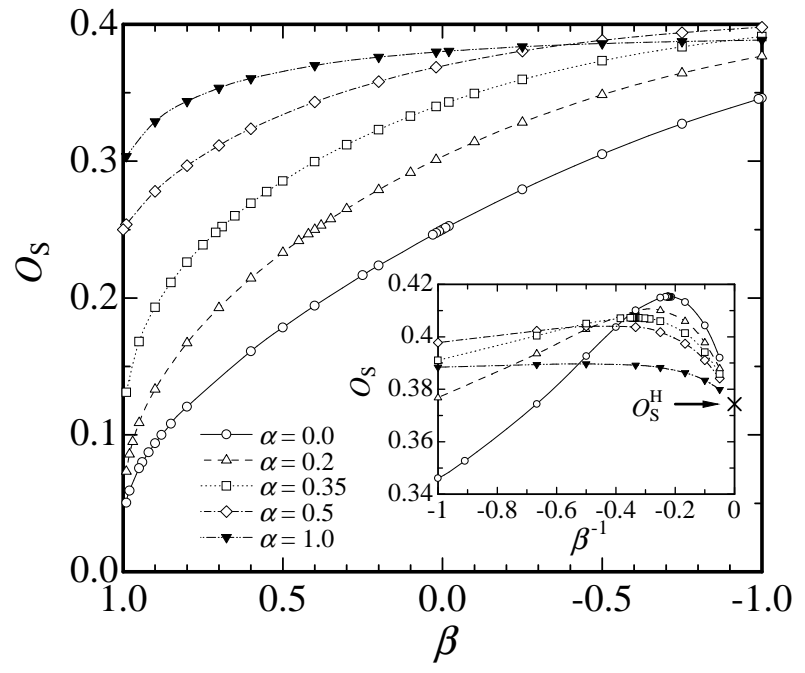

Fig. 8. String order parameter as a function of $\beta(1>\beta \geq-1)$ for some values of $\alpha$. The inset shows the same quantity for $-1>\beta \geq-\infty$. The cross at $\beta^{-1}=0$ indicates $O_{\mathrm{S}}^{\mathrm{H}}(\infty)=$ $0.374325096(2)$ for the $S=1$ Heisenberg model. ${ }^{40)}$ On the SS line $O_{\mathrm{S}}=1 / 4$.

diagram of the IC correlation in the $\alpha$ - $\beta$ plane.

\subsection{Disorder in the real space}

In classical spin systems with competing interactions which favor different wave numbers, e.g. $\pi$ and $\pi / 2$, a transition often occurs from an AF ordered state to spiral ones, as some parameter varies. Corresponding to this transition, in 1D quantum spin systems the oscillatory period of $S_{i j}$ and the wave number which maximizes the static structure factor $S(q)$ change from a commensurate (C) value to an IC one, although LRO's are not realized due to quantum fluctuations. Thus, incommensurability is measured in two ways, namely by $S_{i j}$ and $S(q)$. Generally, the transition point for $S_{i j}$ (disorder point) does not coincides with one for $S(q)$ (Lifshitz point). ${ }^{46)}$ In this subsection, we focus on the former.

Before discussing our results, we briefly review the classification and attribute of C-IC transitions. For Ising-spin systems, various properties of C-IC transitions were studied; the transitions are classified into two kinds. ${ }^{47}$ ) This classification is applicable to the quantum systems. ${ }^{46,48)}$ The disorder point of the first kind has the following properties: In one side of this point $\left|S_{i j}\right|$ has monotonic exponential decay, whereas in the other side the exponential decay is modified by an oscillatory factor with parameter-dependent wavelength, typically as,

$$
\left\langle S_{i}^{z} S_{i+r}^{z}\right\rangle \sim \cos [q(\beta) r] e^{-r / \xi(\beta)} .
$$

In addition, the correlation length $\xi$ behaves in the vicinity of this disorder point $\left(\beta=\beta_{\mathrm{d}}\right)$ as,

$$
\left|\frac{\partial \xi}{\partial \beta}\right|_{\mathrm{C}}=\infty, \quad\left|\frac{\partial \xi}{\partial \beta}\right|_{\mathrm{IC}}=\text { finite, }
$$

where we assume $\beta$ to be the parameter and $\mathrm{C}$ (IC) denotes the limit of $\beta \rightarrow \beta_{\mathrm{d}}$ in the $\mathrm{C}$ (IC) side. On the other hand, the disorder point of the second kind has the 
properties that $S_{i j}$ decays exponentially with parameterindependent oscillations in both sides of the point. Besides, $\xi$ satisfies the following conditions:

$$
\xi=0 \quad\left(\beta=\beta_{\mathrm{d}}\right), \quad\left|\frac{\partial \xi}{\partial \beta}\right|_{ \pm}=\infty
$$

where the double sign indicates the limits from both sides of the disorder point.

Now, we consider our model, which has the competing AF couplings, the nearest-neighbor and NNN terms. As pointed out for Fig. 5(b), there are two C-IC boundaries. The lower boundary coincides with the SS line. Hereafter, we use $\beta_{\mathrm{d}}^{\mathrm{l}}$ and $\beta_{\mathrm{d}}^{\mathrm{u}}$ for the lower and upper boundary, respectively. As for the $\mathrm{C}$ areas, $S_{i j}$ has the $\uparrow \uparrow \downarrow \downarrow$ correlation (period 4) for $\beta<\beta_{\mathrm{d}}^{\mathrm{u}}$, and the AF (staggered) correlation (period 2) for $\beta>\beta_{\mathrm{d}}^{\mathrm{l}}$. In Fig. 9, $\left|S_{i j}\right|$ is shown for some typical IC cases $\left(\beta_{\mathrm{d}}^{\mathrm{u}}<\beta<\beta_{\mathrm{d}}^{\mathrm{l}}\right)$. In contrast to a smooth exponential decay for the $\mathrm{C}$ cases in Fig. 4 , for $\beta>\beta_{\mathrm{d}}^{\mathrm{u}},\left|S_{i j}\right|$ has an oscillation with the long wave length which indicates the discrepancy from the period 4 (i.e. $q=\pi / 2$ ). With increasing $\beta$, this oscillatory period becomes shorter, as seen for $\alpha=1$ in Fig. 9. The inset shows the characteristic wave number $q$ in eq. (4.1), obtained through the following fit:

$$
\left\langle S_{i}^{z} S_{i+r}^{z}\right\rangle e^{\xi / r} \propto \cos [(q+\pi / 2)(r+0.44)] .
$$

The exponent $\theta$ of $q+\pi / 2 \propto\left(\beta-\beta_{\mathrm{d}}^{\mathrm{u}}\right)^{\theta}$ is estimated as $\theta=0.39$ for $\alpha=1$, for example. On the other hand, the condition for $\xi$ eq.(4.2) is also satisfied, e.g. as seen obviously for $(\alpha, \beta)=(1,0.775)$ in Fig. $5(\mathrm{~b})$. Thus, we have found the upper disorder line belongs to the first kind. Through the same procedure for $\beta_{\mathrm{d}}^{\mathrm{l}}$, we have confirmed that the lower boundary also belongs to the disorder point of the first kind. By the way, at the isolated dimer point the upper and lower boundaries merge into one, and the intermediate IC region vanishes [see Figs. 5(b) and 12]. Thus, in both sides of $\beta=0$ the oscillatory periods of $S_{i j}$ are constant (2 and 4), and eq. (4.3) is satisfied as seen in Fig. 5(b). Accordingly, the isolated dimer point belongs to the second kind. In this sense, the C-IC transition of the second kind is regarded as a complex of two transitions of the first kind.

Finally, we summarize our results for three typical cases in Table I with those of the two related $S=1$ spin models for comparison.

\subsection{Incommensurate correlation in $S(q)$}

Another measure of incommensurability is the wave number $q=q_{\max }$ which maximizes the static structure factor:

$$
S(q)=\frac{1}{L} \sum_{i, j}\left\langle S_{i}^{z} S_{j}^{z}\right\rangle e^{i q\left(r_{i}-r_{j}\right)} .
$$

We call $q_{\max }$ as the pitch angle by analogy with the spiral spin order. The C-IC boundary (i.e. Lifshitz point) is defined as the point at which $q_{\max }$ deviates from the $\mathrm{C}$ wave numbers, $\pi$ or $\pi / 2$.

We have estimated $S(q)$ by DMRG under OBC in the

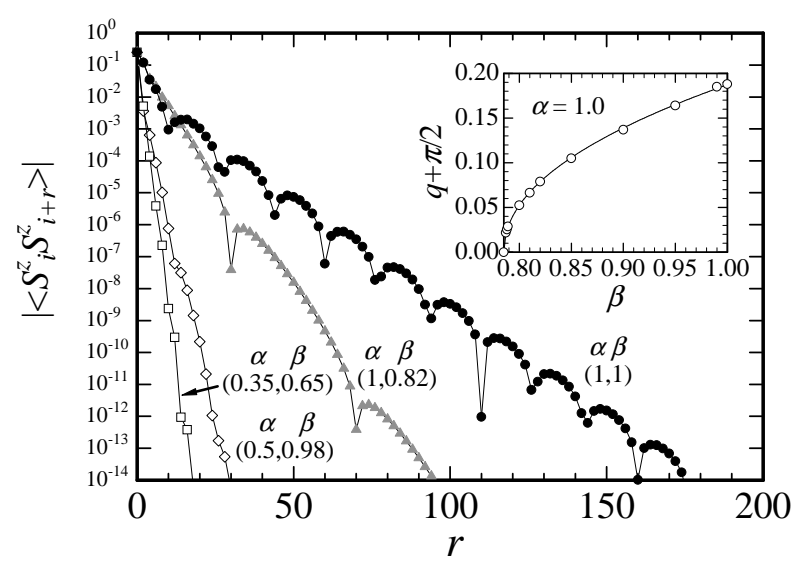

Fig. 9. Spin-spin correlation function as a function of $r$ for some parameter values in the IC area. Only the data of even $r$ are plotted for clarity. The inset shows the characteristic $q$ of eq. (4.1) for $\alpha=1\left(\beta_{\mathrm{d}}^{\mathrm{u}}=0.775\right)$.

following way: Fixing the system size at $L_{0}=280$, we calculate every $S_{i j}=\left\langle S_{i}^{z} S_{j}^{z}\right\rangle$ in the central part of the system. To eliminate the edge effect we do not use 40 sites from both ends, so that $S_{i j}$ 's of $r_{i j}=r_{i}-r_{j} \leq 198$ are obtained. Additionally, thanks to the dimerization, $S_{i j}$ 's of negative $r_{i j}$ can be estimated only by shifting the starting site from $i$ to $i+1$. Therefore, we substantially obtain $S_{i j}$ for the system of $L=2 \times 198$. Since $S_{i j}$ decays exponentially in the region concerned, the long-distance contribution is negligible in the Fourier transformation. The values of $S(q)$ thus obtained coincide with the ED results with $\mathrm{PBC}$ up to $L=26$ within $7-8$ digits.

Figure 10 shows $S(q)$ for various values of $\beta$, and $\alpha=0$. In the $S=1 / 2$ Heisenberg model $(\beta=1), S(q)$ is logarithmically divergent at $q=\pi$ due to the quasilong-range AF correlation. ${ }^{50)}$ At the isolated dimer point $(\beta=0), S(q)=[1-\cos (q)] / 4$. For $1 \geq \beta \geq$ $\beta_{\mathrm{L}}=-0.3723(2), q_{\max }$ remains at $\pi$, and starts to shift from $\pi$ at $\beta_{\mathrm{L}}$ (Lifshitz point). In the Haldane limit $(\beta \rightarrow-\infty), S(q)$ becomes a Lorentzian ${ }^{44}$ sharply peaking at $q_{\max }=\pi / 2$. From the present calculations, however, it is difficult to determine definitely the upper C-IC boundary.

In Fig. 11 we plot $q_{\max }$ for some values of $\alpha$ versus $\beta$. The pitch angle $q_{\max }$ suddenly drops from the higher $\mathrm{C}$ value $\pi$, but slowly approaches the lower $\mathrm{C}$ value $\pi / 2$, as $\beta$ decreases. This aspect is common to the $S=1$ models. ${ }^{48,49)}$ Since for $\alpha>0.52063^{21)} q_{\max }$ is IC even for $\beta=1, q_{\max }$ is always IC for $\alpha=1$. In Fig. 11 also shown is $q_{\max }$ of the static susceptibility $\chi(q)$ for $\alpha=0$, obtained by ED through,

$$
\chi(q)=\int_{0}^{\infty} d \omega \frac{S(q, \omega)}{\omega} .
$$

This quantity is sometimes used to measure the incommensurability, but the pitch angle of $\chi(q)$ does not necessarily coincide with that of $S(q)$.

In this connection, the tendency of IC peak in $S(q)$ has been observed in the inelastic neutron scattering spectrum for doped $\mathrm{CuNb}_{2} \mathrm{O}_{6},{ }^{15)}$ as discussed in Ap- 
Table I. Comparison of the C-IC transition among the three models. The left column is the $S=1$ bilinear-biquadratic Heisenberg chain: $\mathcal{H}=J\left[\sum_{i} \boldsymbol{S}_{i} \cdot \boldsymbol{S}_{i+1}+\gamma \sum_{i}\left(\boldsymbol{S}_{i} \cdot \boldsymbol{S}_{i+1}\right)^{2}\right]$, where $J>0$ and $\gamma>0 .{ }^{46,49)}$ For $\gamma=1 / 3$ the model is so-called AKLT Hamiltonian $^{32}$ ) and is written in the form of a projection operator. The middle column is the $S=1$ frustrated Heisenberg chain: $\mathcal{H}=J\left[\sum_{i} \boldsymbol{S}_{i} \cdot \boldsymbol{S}_{i+1}+\alpha \sum_{i} \boldsymbol{S}_{i} \cdot \boldsymbol{S}_{i+1}\right]$, where $J>0$ and $\alpha>0 .{ }^{48)}$ For the present model we tabulate three typical cases. In the section of $S_{i j}$ the values of the lower and upper disorder points are entered with their classes. In the section of $S(q)$ the values of the lower and upper Lifshitz points are entered with their pitch angles $\left[q_{\max }\right]$ in the $\mathrm{C}$ sides. In the 'Other transition' section we list transitions in the IC regime, if any.

\begin{tabular}{|c|c|c|c|c|c|c|}
\hline & & \multirow{2}{*}{$\begin{array}{c}S=1 \text { bilinear-biquadratic } \\
\text { Heisenberg model }\end{array}$} & \multirow{2}{*}{$\begin{array}{c}S=1 \text { frustrated } \\
\text { Heisenberg model }\end{array}$} & \multicolumn{3}{|c|}{ The present model } \\
\hline & & & & $\alpha$ axis $(\beta=1)$ & $\beta$ axis $(\alpha=0)$ & $\alpha=0.35$ \\
\hline$S_{i j}$ & $\begin{array}{l}\text { lower } \\
\text { upper }\end{array}$ & $\begin{array}{c}\gamma=1 / 3(\text { AKLT }) \\
(\text { first kind) } \\
\gamma=1\end{array}$ & $\begin{array}{c}\alpha=0.284(1) \\
\text { (first kind) } \\
\alpha=\infty ?\end{array}$ & $\begin{array}{c}\alpha=0.5(\mathrm{MG}) \\
\text { (first kind) } \\
\alpha=\infty\end{array}$ & $\begin{array}{c}\beta=0 \\
\text { (second kind) } \\
\beta=0\end{array}$ & $\begin{array}{c}\beta=0.7 \\
(\text { first kind) } \\
\beta=0.445(5) \\
\text { (first kind) }\end{array}$ \\
\hline$S(q)$ & $\begin{array}{l}\text { lower } \\
\text { upper }\end{array}$ & $\begin{array}{c}\gamma=0.43806(4)[\pi] \\
\gamma=1[2 \pi / 3]\end{array}$ & $\begin{array}{c}\alpha=0.373(3)[\pi] \\
\alpha=\infty ?[\pi / 2]\end{array}$ & $\begin{array}{c}\alpha=0.52063(6)[\pi] \\
\alpha=\infty[\pi / 2]\end{array}$ & $\begin{array}{c}\beta=-0.3723(2)[\pi] \\
\beta=\infty ?[\pi / 2]\end{array}$ & $\begin{array}{c}\beta=0.5131(1)[\pi] \\
\beta=\infty ?[\pi / 2]\end{array}$ \\
\hline $\begin{array}{l}\Delta(\mathrm{m} \\
O_{\mathrm{S}}(\mathrm{r}\end{array}$ & $\begin{array}{l}\text { ax) } \\
\text { ax) }\end{array}$ & $\begin{array}{c}\gamma=0.406 \\
\gamma=1 / 3\end{array}$ & $\begin{array}{c}\alpha=0.40(1) \\
\alpha=0.4397(1)\end{array}$ & $\begin{array}{c}\alpha \sim 0.6 \\
\alpha=0.748(2)\end{array}$ & $\begin{array}{c}\beta=0 \\
\beta=-4.47(1)\end{array}$ & $\begin{array}{l}\beta=0.525(5) \\
\beta=-2.93(1)\end{array}$ \\
\hline $\begin{array}{l}\text { Othe } \\
\text { Tran }\end{array}$ & ition & No & $\begin{array}{c}\alpha=0.7444 \\
(\mathrm{AKLT}-\mathrm{NNN} \text { AKLT) }\end{array}$ & No & No & $\mathrm{No}$ \\
\hline
\end{tabular}

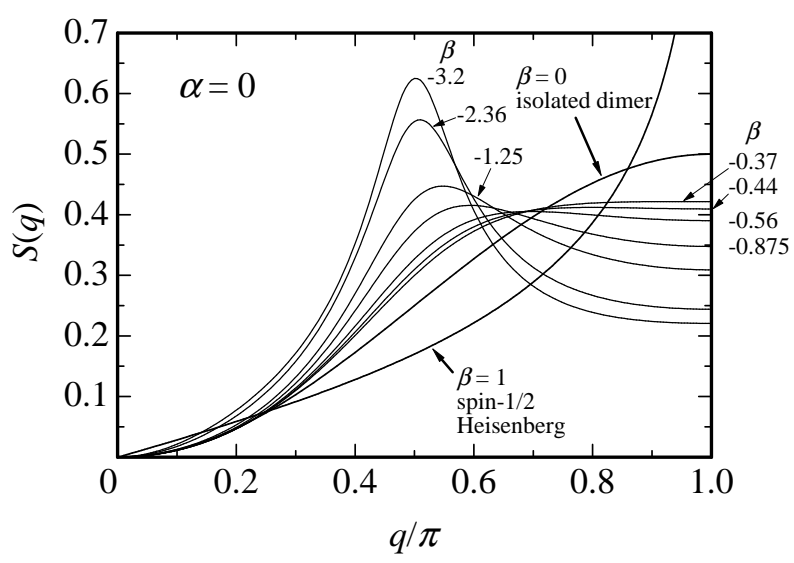

Fig. 10. Static structure factor for various values of $\beta$ and $\alpha=0$. The system used corresponds to the periodic chain of $L=396$ (see text). Note that the line only connects the discrete data points without any interpolation.

pendix D.

\subsection{Phase diagram of incommensurate correlation}

So far, we have studied the IC correlations in terms of three quantities: The wave number of the lowest triplet excitation $E_{\mathrm{t}}(q)$ in $\S 3.1$, the spin-spin correlation $S_{i j}$ in $\S 4.1$ and the static structure factor $S(q)$ in $\S 4.2$. For each quantity we have determined C-IC boundaries in the $\alpha-\beta$ plane, which are depicted in Fig. 12.

The dashed line with triangle represents the boundary between $q=\pi(0)$ and $\pi / 2$ for the lowest $E_{\mathrm{t}}(q)$. On this line the spin gap shows the cusp as seen in Fig. 3. This jump from $\pi(0)$ to $\pi / 2$ with decreasing $\beta$, i.e. cusp, is obvious for $\alpha \lesssim 0.5$, but it disappears for $\alpha \gtrsim 0.5$ due to the IC excitations (see Fig. 24 in Appendix). This line is included in the IC region of $S_{i j}$, and discords from each

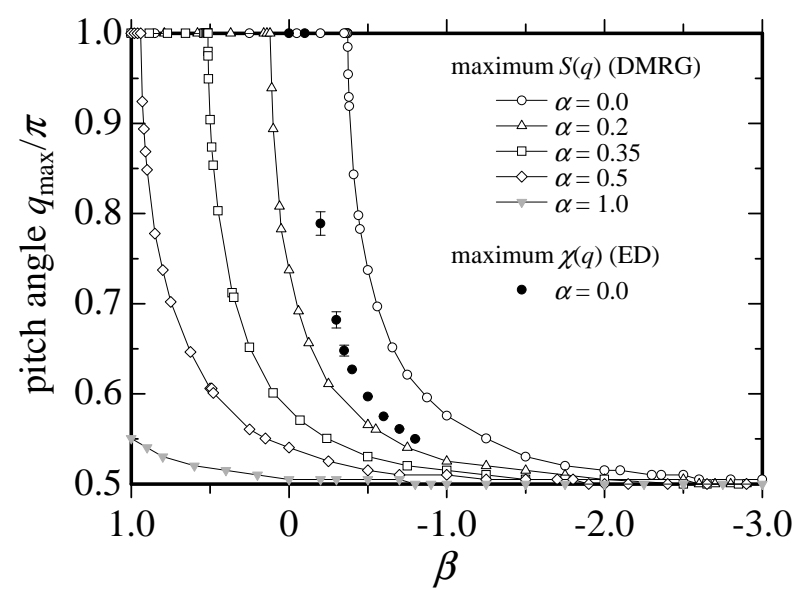

Fig. 11. Pitch angle $q_{\max } / \pi$ of $S(q)$ as a function of $\beta$ for several values of $\alpha$. Since the data are of $L=396$, only discrete values of $q_{\max }$ are allowed. Filled circles shows $q_{\max } / \pi$ of the static susceptibility $\chi(q)$ for $\alpha=0$.

boundary mentioned below.

The shaded area is the IC region where $\left|S_{i j}\right|$ decays exponentially with a quasi-periodic oscillation as in Fig 9 . This region is bounded by the disorder lines indicated by the solid lines with filled circle. The $\mathrm{C}$ region under the lower (over the upper) disorder line has the AF $(\uparrow \uparrow \downarrow \downarrow)$ ) correlation. On the lower boundary (the SS line), the Hamiltonian can be written in the form of projection operators. ${ }^{32)}$ The fact that $\mathcal{H}$ is represented by projection operators at the disorder point is common to some other models. In the spin-orbit model the disorder point is the $\mathrm{SU}(4)$ point,${ }^{51)}$ and in the $S=1$ model the AKLT point, ${ }^{46)}$ as shown in Table I.

The thin solid lines show the contours of the pitch angle $q_{\max } / \pi$, which maximizes $S(q)$. In contrast to other 
cases, ${ }^{46,48)}$ the IC region of $S(q)$ extends widely in the $\mathrm{C}$ region of $S_{i j}$. The pitch angle decreases abruptly near the Lifshitz line $\left(q_{\max }=\pi\right)$. In such a region $S(q)$ shows merely a broad hump instead of a sharp peak (Fig. 10), hence $q_{\max }$ is hardly regarded as a characteristic wave number there. Indeed, we have not observed a singular behavior in any quantity at the Lifshitz point; systemsize dependence of many quantities in this IC region shows the period of 4 , in accordance with the $\mathrm{C}$ period of $S_{i j}$. Thus, the intrinsic IC correlation is represented in terms of $S_{i j}$.

In Fig. 12 all the lines go down rightward. As repeatedly mentioned, this indicates that the properties are preserved along this direction due to the similar role of $-\beta$ and $\alpha$.

Lastly, we touch on the continuity of the Haldane phase. The IC regime lies between the Haldane limit $(\beta=-\infty)$ and $\beta=1$ irrespective of the value of $\alpha$. Indeed, the correlation length and the spin gap are singular there. However, various quantities including the dimer and string order parameters (§3.3), which characterize the Haldane phase, have no anomaly. Actually, we will confirm that the Haldane phase extends to the limit of $\beta \rightarrow 1$ in $\$ 5$ and Appendix C. Hence, the IC correlation does not affect the essence of the Haldane phase and also of the spin liquid phase.

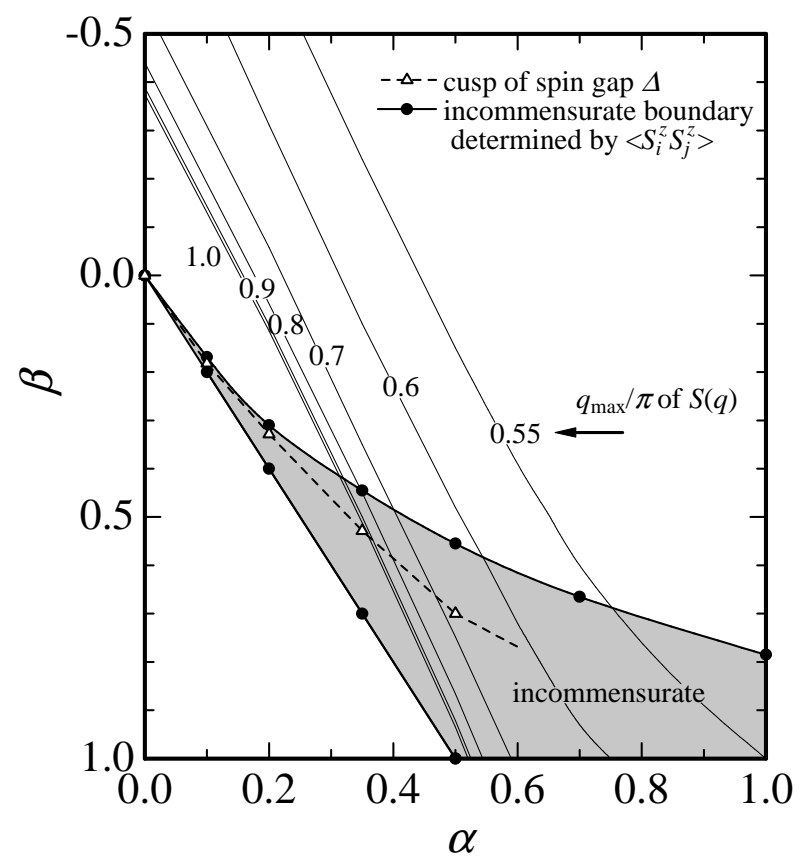

Fig. 12. Comprehensive phase diagram of IC correlation in eq. (1.1). Shaded area is the IC regime in $S_{i j}$. Thin solid lines are the contours of pitch angle $q_{\max }$ which maximizes $S(q)$; the Lifshitz line corresponds to the line of $q_{\max } / \pi=1.0$. The IC area in $S(q)$ extends further in the directions of $\alpha \rightarrow \infty$ and $\beta \rightarrow-\infty$. Dashed line with open triangle shows the cusp of the spin gap, which distinguishes the wave numbers of the lowestenergy excitation between $q=\pi$ and $\pi / 2$ (see $\S 3.1$ ).

\section{§5. Spin-Peierls Regime}

In this section, we concentrate on the Spin-Peierls regime. First, the low-lying excitation is reconsidered in $§ 5.1$. Next, we study the edge effects in the light of the delocalized spin polarization, and consider the origin of the impurity effect of $\mathrm{CuGeO}_{3}$ in $\S 5.2$. The stability of the edges is considered in reference to the lattice distortion in spin-Peierls compounds in $§ 5.3$. The NMR spectra is discussed in $§ 5.4$.

\subsection{Two-spinon bound state}

One primitive modeling of the substitution by nonmagnetic elements is to consider finite-size systems, assuming that the impurities simply cut the $1 \mathrm{D}$ chain into finite segments. For finite systems, the boundary conditions are essentially important, in particular for the dimerized cases $(\beta<1)$. Then, there are two kinds of exchange coupling at the chain ends: $J$ (stronger one) or $\beta J$ (weaker one). For simplicity, we call the former (latter) case as the strong (weak) edge. In even-site systems both of the edge couplings are $J$ or $\beta J$.

The local spin polarization $\left\langle S_{i}^{z}\right\rangle$ is an important quantity to study the local properties of magnetic excitation and the tendency toward magnetic LRO's. ${ }^{52)}$ Figure 13 shows $\left\langle S_{i}^{z}\right\rangle$ of the lowest-energy state in the $S^{z}=-1$ subspace for $\alpha=0$ (I) and $\alpha=0.35$ (II). For both values of $\alpha$ the cases of the strong and weak edge are shown in (a) and (b), respectively. As described in Appendix C.2, the strong edge has some analogous properties to $\mathrm{PBC}$ or to the bulk. Since the main subject of this subsection is the low-lying excitation in the bulk, we restrict our discussions to the qualitative feature of the strong edge, here. We will concentrate on the edge properties and impurity effect in $§ 5.2-\S 5.4$.

In Figs. $13 \mathrm{I}(\mathrm{a})$ and II(a) there are two humps of staggered moment, and the amplitude becomes maximum for $\beta=1$. These humps are considered as the amplitude of excited free spinons $\left(S=1 / 2\right.$ entity). ${ }^{53,56)}$ For the $S=1 / 2$ Heisenberg model $[(\alpha, \beta)=(0,1)]$, it is known that the excitation can be exactly described by the scattering of even number spinons. ${ }^{57,58)}$ For the MG point $[(\alpha, \beta)=(0.5,1)]$, a similar free spinon picture was successfully introduced from a variational viewpoint, ${ }^{24)}$ and recently it was numerically shown that the MG gap (twospinon excitation) is accurately twice the bottom of the single spinon blanch for odd-site systems. ${ }^{53)}$ Thus, the free-spinon picture seems applicable to all the values of $\alpha$ for $\beta=1$ at least in the low-lying excitation. When the dimerization is switched on $(\beta<1)$, the amplitude of the oscillating part is abruptly reduced, and almost disappears for $\beta=0.2$ leaving a broad peak as seen in Figs. $13 \mathrm{I}(\mathrm{a})$ and $\mathrm{II}(\mathrm{a})$. The mechanism of this process has been pursued by many studies. ${ }^{18,53,59-61)}$ When $\beta$ decreases from 1 , two spinons are bound to make a magnon ( $S=1$ entity) described by a single parameter; an isolated blanch thereby appears below the continuum. Furthermore, multi-magnon branches have been found above it. ${ }^{13,53,62)}$

To grasp intuitively the process from spinon to magnon, it is useful to consider the excitation along the 
Fig. 13. Local spin polarization of the lowest-energy state in the subspace of $S^{z}=-1$ for open systems with $L=200$ as a function of $i$ for $\alpha=0$ (I) and $\alpha=0.35$ (II). At both chain ends the strong coupling $J$ is assumed in (a), while the weak coupling $\beta J$ in (b). For $\beta \sim 1$ in every panel, $\left\langle S_{i}^{z}\right\rangle$ oscillates alternately with considerable amplitude.

SS line. At the MG point the ground state is twofold degenerate, $\psi_{1}$ and $\psi_{2}$ in eq. (2.1). Here, we call the singlet array of $\psi_{1}\left(\psi_{2}\right)$ as the $\mathbf{R}(\mathrm{L})$ array [Figs. $14 \mathrm{I}(\mathrm{a})$ and $\mathrm{I}(\mathrm{b})$ ]. In the lowest-energy excitation a singlet pair breaks into two spins (spinons), which sit on the mutually different subchains (legs) to avoid breaking another singlet pair, as shown in Fig. 14 II. The resultant spinons become two domain walls, between which the phase of singlet array, say L, is opposite to the original one, R. In this case the $\mathrm{L}$ array has an equivalent energy to the $\mathrm{R}$ array, so that the spinons move freely regardless of the distance between them. Thus, these scattering states make a low-energy continuum. On the other hand, when $\beta$ is reduced from 1 keeping $\beta=2 \alpha$, the degeneracy of the ground state is lifted. One of the states, say $\psi_{1}$ in which singlets are positioned on the strong bonds $(J)$ is still the exact ground state for $\beta<1$, while $\psi_{2}$ is no longer an eigenstate and even has high energy over the singlet-triplet gap, as explained in Appendix C. Consequently, the L array is energetically unfavorable and works as a linear confinement potential; the two spinons approach each other to reduce the region of L. Namely, the configuration of Fig. 14 III(b) is more likely than that of Fig. 14 III(a). The resultant bound state causes an isolated branch below the continuum. Although we have focused on the SS line, this explanation is qualitatively valid for all the other paths from the $\alpha$ axis. This change of the excitation mechanism corresponds to the transi- tion from the spin liquid phase to the Haldane phase, ${ }^{18)}$ and is analogous to the so-called $\Delta$ chain. ${ }^{63)}$

Actually, one can confirm the appearance of the isolated magnon branch in the dynamical structure factor,

$$
S(q, \omega)=\sum_{n}\left|\left\langle\Psi_{n}\left|S_{q}^{z}\right| \Psi_{0}\right\rangle\right|^{2} \delta\left(\omega-\left(E_{n}-E_{0}\right)\right),
$$

where $\hbar=1, \Psi_{n}\left(E_{n}\right)$ is the $n$-th eigenfunction (eigenvalue) of the system, and the ground state is indicated by $n=0 . \quad n$ runs over all the eigenstates. $S(q, \omega)$ is proportional to the intensity of inelastic neutron scattering spectra. In Figs. 15(a)-(c) we show $S(q, \omega)$ for $\tilde{\alpha}=0.35$, which is the plausible value for $\mathrm{CuGeO}_{3} \cdot{ }^{64)} \mathrm{As}$ discussed in the previous paper, ${ }^{13)}$ the lowest mode for $\delta=0(\beta=1)$ [Fig. 15(a)] is assigned to the continuum by checking the system-size dependence of the residue and position of pole in $S(q, \omega)$. As soon as the dimerization is introduced, weak intensity appears below the continuum, whose position is almost independent of $L$, as seen in Fig. 15(b). This branch remarkably grows when $\delta$ increases to $0.012(\beta=0.98)$ [Fig. 15(c)], which is an expected value for $\mathrm{CuGeO}_{3}$ within eq. (1.1). ${ }^{61,62)}$ This abrupt increase of the intensity is obvious in the inset of Fig. 15(b), where the ratio of intensity (residue) for the lowest branch at $q=\pi / 2$ is plotted. Since the intensity belonging to the continuum decreases with $1 / L$ as $L \rightarrow \infty$, this isolated magnon branch makes a dominant contribution. 
Fig. 15. Dynamical structure factor $S(q, \omega)$ for three values of $\delta$ are shown for the fixed value of $\tilde{\alpha}=0.35$. Data obtained by the ED with a recursion method for $L=14-26$ are simultaneously plotted. The intensity is proportional to the area of circle. The magnon branch is indicated by arrow in (b) and (c). The inset in (b) shows the intensity ratio (residue) of the lowest branch at $q=\pi / 2$ versus $\delta$ for $L=20$ and 24. System-size dependence is small in this wave number.

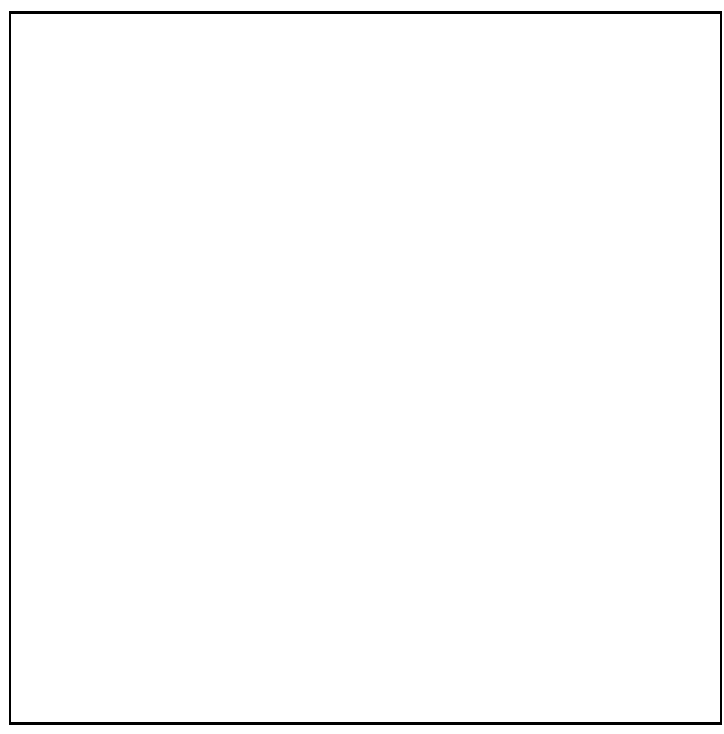

Fig. 14. Schematic figures for low-energy excitations along the SS line $(\beta=2 \alpha)$. I(a),(b): The degenerate ground states for the MG point. II: The lowest-excited state at the MG point, in which two spinons on different subchains (legs) form domain walls. III(a),(b): Excited states on the SS line $(\beta<1)$, where two spinons are located apart and closely, respectively. See text for details.

Recent neutron experiments ${ }^{66)}$ for $\mathrm{CuGeO}_{3}$ vividly showed the appearance of the magnon branch. In the measurement at $T=12 \mathrm{~K}$ (immediately below $T_{\mathrm{SP}} \sim$
$14 \mathrm{~K})$ the spectrum has a sharp peak below the continuum as previously observed, ${ }^{67)}$ whereas at $T=16 \mathrm{~K}$ this sharp peak suddenly vanishes and only a broad continuum is observed. Thus, the change in the excitation mechanism between $\delta=0$ and $\delta>0$ has been confirmed by experiment.

Lastly, we touch on the thermodynamics. According to the abrupt change of the level structure near $\delta=0$, thermodynamic quantities drastically changes, too. We confirmed that by calculating the specific heat $C(T)$ along the SS line. As soon as $\tilde{\alpha}$ decreases from 0.5, a sharp peak appears. This peak is attributed to the dense low-lying triplet levels caused by the dimerization. The situation is the same with the $\Delta$ chain. ${ }^{63)}$

\subsection{Delocalized spin polarization}

In this subsection, we study $\left\langle S_{i}^{z}\right\rangle$ in the lowest magnetic state $(S \neq 0)$ to clarify the impurity effect. We assume that non-magnetic impurities cut the chain at random into odd- and even-site segments. This assumption is not so crude, since the NNN exchange hardly change the situation, as will be discussed later.

First, we look at the odd-site systems. Although for even-site segments the ground state is always singlet and $\left\langle S_{i}^{z}\right\rangle=0$ for each $i$, yet for odd-site segments the ground state is $S=1 / 2$, therefore $\left\langle S_{i}^{z}\right\rangle \neq 0$. In Fig. 16(a) we show $\left\langle S_{i}^{z}\right\rangle$ for $L=21$ and $\alpha=0.35$ in the $S^{z}=1 / 2$ sector. For the non-dimerizing case $(\beta=1)$, the spin polarization extends to the whole system, corresponding to a spinon discussed in $\S 5.1$. This delocalized spin polarization is still seen in the IC regime $(\alpha>0.5)$ as far as $\beta=1$, as shown in Fig. 16(b). Therefore the delocal- 
ized spin polarization is characteristic of the spin-liquid phase, and has nothing to do with the existence of a gap. For $\beta<1$ one of the chain ends is the strong edge $[i=21-22$ in Fig. 16(a)] and the other the weak edge $(i=1-2)$. With decreasing $\beta$, the magnetic moment becomes localized near the weak edge and the amplitude near the strong edge vanishes. Here, let us return to Figs. $13 \mathrm{I}(\mathrm{b})$ and $\mathrm{II}(\mathrm{b})$, where the lowest excited states $(S=1)$ in even-site segments with the weak edges are shown. These states are obviously regarded as the duplication of the ground state of odd-site systems $(S=1 / 2)$ connected at the strong edges. Hence, the localization of magnetic polarization is a characteristic of the weak edge. This free $S=1 / 2$ excitation at the edge is nothing but the celebrated aspect of the Haldane chain. ${ }^{33,68)}$

In the following, we treat this localized moment more quantitatively. Consider a finite system with $L$ spins, as in Figs. $13 \mathrm{I}(\mathrm{b})$ and $\mathrm{II}(\mathrm{b})$. For the non-dimerized case $(\beta=1)$, the range of finite $\left\langle S_{i}^{z}\right\rangle$ extends to the whole chain irrespective of the value of $\alpha$, as mentioned. When dimerization is introduced, this range shrinks toward the edges. Incidentally, in the spin-Peierls regime $(\beta \sim 1)$, $\left\langle S_{i}^{z}\right\rangle$ around the edges does not obey the simple exponential decay unlike the Haldane regime, so that the localization length is not an appropriate measure of the localization range. Therefore, we introduce a somewhat artificial quantity $\eta_{\text {edge }}$, defined as the maximum integer satisfying $\left|\left\langle S_{i}^{z}\right\rangle\right|>10^{-3}$ for all $i<\eta_{\text {edge. }}$ Here, we choose the value $10^{-3}$ merely for convenience. In Fig. 17 we show $\eta_{\text {edge }}$ estimated for the systems of $L=200$. Note that $\eta_{\text {edge }}$ scarcely depends on the system size, in contrast to $\left\langle S_{i}^{z}\right\rangle$ for the strong edge. The localization range abruptly becomes narrow with decreasing $\beta$ from 1. With increasing $\alpha, \eta_{\text {edge }}$ behaves more abruptly. For $\beta \lesssim 0.9,\left\langle S_{i}^{z}\right\rangle$ around the edges shows the typical exponential decay characteristic of the Haldane phase.

Next, returning to the Figs. 13 I(a) and II(a), we give quantitative treatment of the strong edge. In this case the local moment can be decomposed into the uniform and the staggered parts as, ${ }^{54,55)}$

$$
\left\langle S_{i}^{z}\right\rangle=\left\langle S_{i}^{z}\right\rangle^{\mathrm{u}}+(-1)^{i}\left\langle S_{i}^{z}\right\rangle^{\mathrm{s}} .
$$

For $\alpha=0$ the uniform part $\left\langle S_{i}^{z}\right\rangle^{\mathrm{u}}$ is almost constant especially in the bulk for $\beta=1 ;\left\langle S_{i}^{z}\right\rangle^{\mathrm{u}}$ abruptly deforms with decreasing $\beta$, and converges to a sinusoidal curve for $\beta \rightarrow 0$. For instance, see $\beta=0.2$ in Figs. $13 \mathrm{I}(\mathrm{a})$. Since the amplitude of the curve is determined by the magnetization and the system size, $\left\langle S_{i}^{z}\right\rangle^{\mathrm{u}} \propto 1 / L$ as shown in the inset of Fig. 17. On the other hand, the staggered part $\left\langle S_{i}^{z}\right\rangle^{\mathrm{s}}$ is drastically suppressed with decreasing $\beta$ and vanishes at $\beta=0$, as shown in Fig. 17. Furthermore, it is important that $\left\langle S_{i}^{z}\right\rangle^{\mathrm{s}}$ highly depends on the system size, and vanishes as $L \rightarrow \infty$ (inset of Fig. 17). Thus, $\left\langle S_{i}^{z}\right\rangle \sim\left\langle S_{i}^{z}\right\rangle^{\mathrm{u}}$ for $\beta \sim 0$. The behaviors of $\left\langle S_{i}^{z}\right\rangle$ is basically the same for $\alpha=0.35$.

Based on the above results, we consider the impurity effects in the spin-Peierls regime, especially with $\mathrm{CuGeO}_{3}\left[\alpha=0.35, \beta=0.97-0.98\right.$ for $\left.T<T_{\mathrm{SP}}\right]$ in mind. For pure $\mathrm{CuGeO}_{3}$ a magnetic LRO has never been observed, while for a doped sample with $0.12 \%$ of $\mathrm{Zn}$ shows

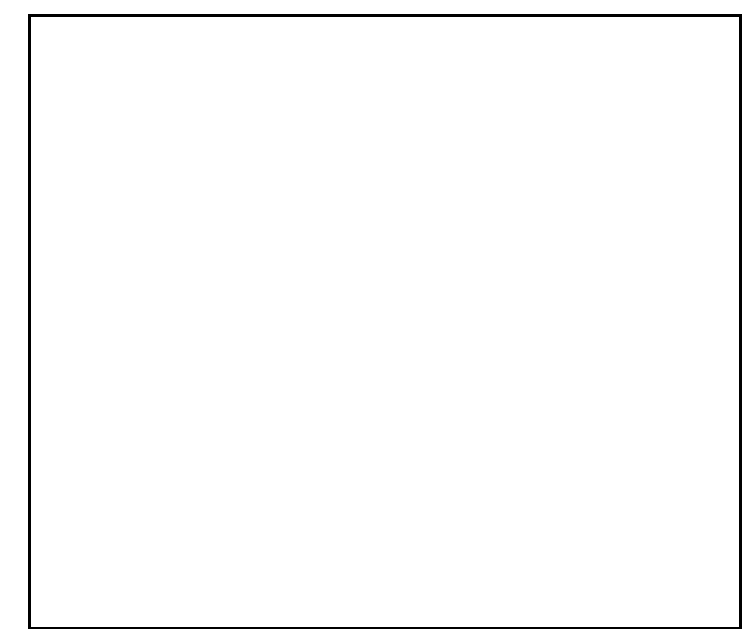

Fig. 16. Local spin polarization in the ground state $\left(S^{z}=1 / 2\right)$ for various cases. (a) Segment with odd sites for some values of $\beta$. A weak edge $(\beta J)$ is assumed in the left chain end. (b) Segment with $L=21$ in the IC regime without the coupling alternation. (c) System with $L=24$ in which the 12 th site is replaced by a site impurity. See text for details. In the margin of (a) and (c), the respective connectivity is schematically shown. As for (b), the connectivity is the same with (a).

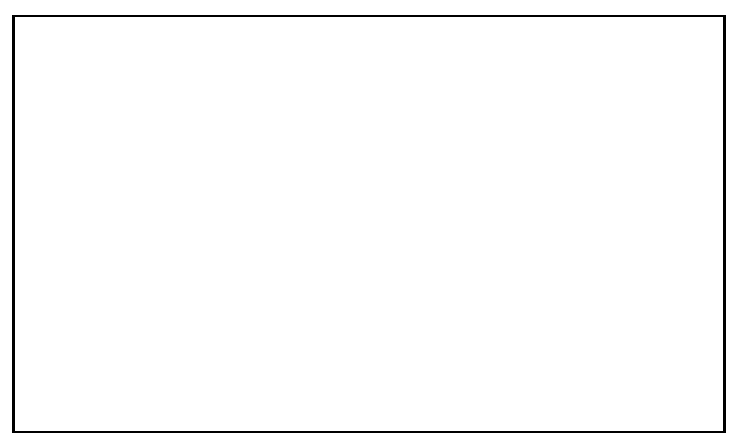

Fig. 17. Amplitude (maximum value) of $\left\langle S_{i}^{z}\right\rangle^{\mathrm{s}}$ for the strong edge, and the range of localized moment near the chain ends $\eta_{\text {edge }}$ for the weak edge, as a function of $\beta$. The inset shows the system-size dependence of the uniform part (value at the midchain site) and the staggered part (maximum value) of $\left\langle S_{i}^{z}\right\rangle$ for the Heisenberg model. The used states have the lowest energies in the $S^{z}=-1$ subspace.

AFLRO in the low temperature. ${ }^{4)}$

[1] Infinite systems $(L=\infty$, pure material) belong to the Haldane phase, as discussed in Appendix C. (a) For the open strong edge, the staggered polarization extends to the whole chain, however its amplitude becomes infinitesimally small as $L \rightarrow \infty$, as shown in Fig.17. Thus a magnetic LRO will hardly arise, even if the interchain coupling is somewhat large. (b) For the open weak edge, there appears staggered polarization with considerably large amplitude in the range of about 30 sites around the edges, but this range is negligible against $L=\infty$. 
The bulk area is not affected by this moment; the higher dimensional coupling will hardly induce a magnetic LRO also in this case. Thus, regardless of the kind of edge, infinite (pure) systems will keep the gap properties of the Haldane phase (see $\S 6$ ).

[2] Finite systems (impurity-doped material) may break the characteristics of the Haldane phase (Appendix C). (a) For the open strong edge, the amplitude of $\left\langle S_{i}^{z}\right\rangle^{\mathrm{s}}$ in the whole system increases with the system size decreasing, in particular abruptly for the thin impurity concentration (inset of Fig. 17). Thus, making use of the enhanced staggered moment, the interchain coupling probably induce AFLRO. (b) For the open weak edge, with decreasing the system size, the bulk part with $\left\langle S_{i}^{z}\right\rangle=0$ vanishes, since $\eta_{\text {edge }}$ is independent of $L$. As a result, the localized polarization around the edges extends to the whole system. AFLRO is likely to appear also in this case.

To summarize, when the system becomes finite by doped with non-magnetic impurities, the properties of the spin liquid are brought about to the spin-Peierls regime, which originally belongs to the Haldane phase for the pure case, and AFLRO will be induced by the interchain magnetic coupling. The above results are not qualitatively influenced by the value of $\alpha$, so that the same mechanism works for spin-Peierls materials in general. So far, we have not made allowance for the stability of the edges. In fact, the strong edge is always stable against the weak edge in the spin-Peierls regime. We will discuss this point in the next subsection.

In this connection, recent $\mu \mathrm{SR}$ measurements for doped $\mathrm{CuGeO}_{3}$ have observed the spatial inhomogeneity of the ordered moment size. ${ }^{69)}$ However, its local structure has not been elucidated yet. Meanwhile, concerning $\left\langle S_{i}^{z}\right\rangle$, the result of the phase Hamiltonian approach ${ }^{70)}$ is similar to the weak-edge case, if anything.

Finally, we mention the effect of the NNN coupling across the impurity. Consider a system consisting of 24 sites but the spin on the 12 th site is replaced by a site impurity like $\mathrm{Zn}$ in $\mathrm{CuGeO}_{3}$ [Fig. 16(c)]. Thereby, the system is separated into odd-site (11) and even-site (12) segments by the impurity, but the NNN exchange $(\alpha=0.35)$ connects them. Since this system has odd spins (23), the ground state is magnetic $(S=1 / 2)$. In Fig. 16(c) we shows a typical result among various coupling conditions calculated. Here, we set the weak edge in the odd-site segment next to the impurity (between $i=10$ and 11) and the strong edges for the even-site segment. An important aspect of this result is that the large amplitude of $\left\langle S_{i}^{z}\right\rangle$ in the odd-site segment scarcely extends to the even-site segment beyond the impurity. This aspect does not change under other coupling conditions and also in the cases of a bond impurity like Si in $\mathrm{CuGeO}_{3}$. Summing up, the NNN coupling does not severely affect our simple treatment of the impurity effect.

\subsection{Stability of the edges}

An important factor for the impurity effect in spinPeierls compounds is the softness of the lattice. In the low temperature the lattice distorts so as to minimize the sum of the lattice distortion energy and magnetic one. We can allow for this factor to some extent by considering the stability among different edge conditions.

First, we consider the energy difference between the ground states of the strong and the weak edges in evensite systems. In Fig. 18(a) we plot

$$
\Delta E(L)= \begin{cases}E(\mathrm{SS}, L)-E(\mathrm{WW}, L), & \text { even } L \\ E(\mathrm{SS}, L)-E(\mathrm{SW}, L), & \text { odd } L\end{cases}
$$

versus $\delta$ for $\tilde{\alpha}=0.35$. Here, $E(\mathrm{SS}, L)$ means the groundstate energy of the system with $L$ sites and the strong edges [cf. Fig. 18(b)]. In this range of $\delta$ the energy of the strong edges is always lower than that of the weak edges. The behaviors of $E(\mathrm{SS}, L)$ and $E(\mathrm{WW}, L)$ are identical with those along the SS line. Namely, $E(\mathrm{SS}, L)$ is a monotonically decreasing function of $\delta$ and proportional to $L$, whereas $E(\mathrm{WW}, L)$ highly depends on $L$ for $\delta \sim 0$, and has discontinuity at $\delta=0$ for $L \rightarrow \infty$. Therefore, $\Delta E(\infty)$ in the limit of $\delta \rightarrow 0$ is finite and equivalent to the gap at $\delta=0$. For details, refer to Appendix C. The finite $\Delta E(\infty)$ for $\delta \rightarrow 0$ in this case is recognized in the inset, where $\partial \Delta E / \partial \delta$ diverges for $L \rightarrow \infty$. Next, for odd $L$ we also plot $\Delta E(L)$ of eq. (5.3) in Fig. 18(a). Here, $E(\mathrm{SW}, L)$ is the ground-state energy of regular odd chain, while $E(\mathrm{SS}, L)$ is that of the strong edges at both chain ends [See Fig. 18(b)]. The defect (irregular pattern) of exchange coupling arising in the latter is placed at (or near) the center of the chain. Since $\Delta E$ is negative even in this case, the strong edge lowers the energy, overcoming the energy loss by the defect. Incidentally, $E(\mathrm{WW}, L)$ is much higher than both energies above. Furthermore, concerning the first excited (triplet) state, we compare the various cases of Figs. 13(a) and (b) $(L=200)$. Also in these cases the strong edge has lower energy than the weak edge.

Thus, we conclude that in the spin-Peierls regime both energies of the ground state and of the lowest excited state are stabilized by the strong edges. In other words, if the lattice is sufficiently flexible like spin-Peierls compounds, the edge becomes strong. We would like to emphasize that this conclusion holds even when the elastic energy of the 3D lattice is included. Because, as is known, the decrement of magnetic energy due to the spin-Peierls distortion $E_{\text {mag }} \propto \delta^{\nu}(\nu \leq 4 / 3)$ overcomes the increment of elastic energy of the lattice $E_{\text {lat }} \propto \delta^{2}$ for $\delta \rightarrow 0 .{ }^{13,71)}$

The result that the strong edge is favorable is consistent with the enhancement of singlet correlation at the edge bond, ${ }^{54,63)}$ although this is not the case for large negative values of $\beta$, as will be considered in $\S 6$. In the above we have discussed simply with fixed-frame lattices. To treat the spin-Peierls transition more accurately, one needs to introduce the magneto-elastic coupling. However, the results of recent studies ${ }^{55)}$ allowing for such a factor are basically the same with ours.

Based on the above results, we speculate on the firstorder compositional phase transition in $\mathrm{CuGeO}_{3}$ in the remainder of this subsection. Recently, Masuda et al. ${ }^{72)}$ reported that when non-magnetic impurity $\mathrm{Mg}$ is doped as $\mathrm{Cu}_{1-x} \mathrm{Mg}_{x} \mathrm{GeO}_{3}$, a clear first-order transition is ob- 


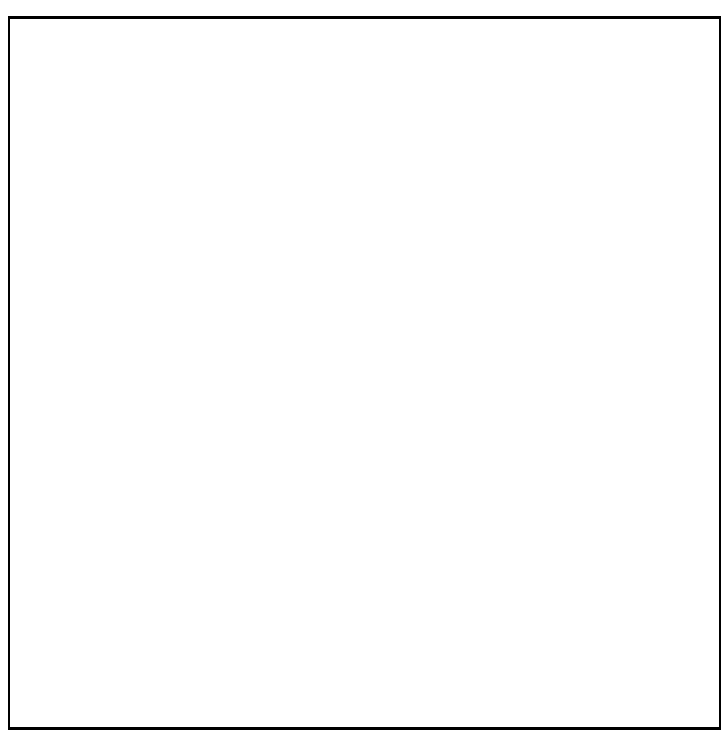

Fig. 18. (a) Energy difference of the ground state between the two edge conditions, which are different between the even and odd segments, as shown schematically in (b). For details see text. In the inset of (a) plotted is the inverse of $J \partial \Delta E / \partial \delta$ estimated by small values of $\delta$.

served at $x=x_{\mathrm{c}} \sim 0.023$ (later modified as $0.027^{73)}$ ) between the dimerized and uniform AF ordered phases. The dependence of $T_{\mathrm{N}}$ on $x$ has a discontinuity at $x_{\mathrm{c}}$ and the spin-Peierls order disappears for $x>x_{\mathrm{c}}$. A recent neutron scattering study ${ }^{73}$ ) has supported this first-order transition. According to it, the amplitude of magnetic moment $\mu_{\text {eff }}$ as well as that of the spin-Peierls distortion have jumps at $x_{\mathrm{c}}$.

For $T<T_{\mathrm{SP}}$ the lattice of $\mathrm{CuGeO}_{3}$ distorts in every direction of the three crystal axes ${ }^{74}$ ) (The $c$ axis is the chain direction). The array of bond alternation in a chain is "out of phase" against those in the adjacent chains in both a- and b-axis directions, namely -L-R-L-R- in the notation of Fig. 14 I. When a non-magnetic impurity cuts the chain, the two edge bonds next to the impurity have to be strong. Thus, the array in one side of the impurity becomes "in phase" locally, e.g. -L-L-L-. This "in-phase" array raises the elastic energy, so that this reversed phase should be relaxed to the original "outof-phase" array in some distance from the impurity. ${ }^{11)}$ Actually, it is observed that the width of spin-Peierls superlattice peaks in the neutron scattering spectra shows the resolution limit, ${ }^{75}$ ) namely the intrachain phase of dimerization does not change globally across the impurity.

When the concentration of impurity is thin, the contribution of the above local defects to the total energy is smaller than the energy gain by the global spin-Peierls distortion. As $x$ increases, local defects appear here and there, which damage the latter energy gain. Eventually, for $x>x_{\mathrm{c}}$ the contribution of the defects overcomes the energy reduction by the global dimerization. Thus, the compositional transition of the first order may be understood by the competition between the 3D global spin-Peierls distortion and the $1 \mathrm{D}$ local defects. ${ }^{76)}$ The discontinuous increase of $\mu_{\mathrm{eff}}$ at $x=x_{\mathrm{c}}$ is understood naturally; if the dimerization vanishes by the above firstorder transition, $\left\langle S_{i}^{z}\right\rangle$ is enhanced discontinuously. For instance, imagine that $\beta$ jumps from 0.99 to 1.0 in Fig. 13 II(a).

\subsection{NMR spectra}

In this subsection we discuss the impurity effect on the NMR spectrum $g(H)$. Since non-magnetic states $(S=0)$ do not contribute to the local susceptibility,

$$
\chi_{i}=\frac{1}{T} \sum_{j}\left\langle S_{i}^{z} S_{j}^{z}\right\rangle_{T},
$$

$\chi_{i}$ is proportional to $\left\langle S_{i}^{z}\right\rangle$ with respect to the lowest magnetic state in the $T \rightarrow 0$ limit. In eq. (5.4) $\langle\cdots\rangle_{T}$ indicates the thermal average. Then, by replacing $\chi_{i}(T)$ by $\left\langle S_{i}^{z}\right\rangle$ the NMR spectra is expressed as

$$
g(\tilde{H})=\sum_{i} f\left[\tilde{H}-\left\langle S_{i}^{z}\right\rangle\right]
$$

where $f(H)$ is the NMR line shape with natural line width at each site, and we assume $f(H)=\exp \left(-H^{2} / w^{2}\right)$ in this paper. And $\tilde{H}=H /\left(A g \mu_{\mathrm{B}}\right)$ with $A$ being the hyperfine coupling of the material. Like eq. (5.2), local susceptibility is sometimes decomposed into the uniform and staggered parts:

$$
\chi_{i}=\chi_{i}^{\mathrm{u}}+(-1)^{i} \chi_{i}^{\mathrm{s}} .
$$

When $\chi_{i}^{\mathrm{u}}$ is independent of $i$, it is nothing but the bulk susceptibility $\chi=\chi_{i}^{\mathrm{u}}$ and only shifts $g(H)$. Actually, $\chi_{i}^{\mathrm{u}}$ often depends weakly on $i$ as in Fig. 13(a). On the other hand, $\chi_{i}^{\mathrm{s}}$ determines the line shape. When $\chi_{i}^{\mathrm{s}}$ has a different oscillatory period from $\pi, e . g$. for the IC or $\uparrow \uparrow \downarrow \downarrow$ structure, the decomposition of eq. (5.6) is unavailable.

First, we look at the Heisenberg model $(\alpha=0, \beta=1)$. Eggert and Affleck have obtained $\chi_{i}^{\mathrm{s}}(T)$ for OBC by the conformal field theory: ${ }^{77}$ )

$$
\chi_{i}^{\mathrm{s}}(T)=\frac{2 a}{\pi} \frac{i}{\sqrt{(J / 2 T) \sinh (4 T i / J)}},
$$

with a constant $a \sim 0.58$. The chain end is at $i=0$. In relatively high temperatures $\chi_{i}$ has finite amplitude only near the chain end, whereas with decreasing temperature the oscillation extends to the inner part of the chain and its amplitude increases. This aspect is consistent with the delocalization of $\left\langle S_{i}^{z}\right\rangle$ for $T \rightarrow 0$ and $\beta=1$. Those changes cause the broadenings of NMR line shape and of background intensity with sharp edges, respectively. Such behaviors were actually observed by an NMR experiment for a good Heisenberg compound $\mathrm{Sr}_{2} \mathrm{CuO}_{3} \cdot{ }^{78)}$

Since eq. (5.7) is the result for the infinite system, we consider finite-size effects. In Figs. 19 I and II we show $g(\tilde{H})$ for $\alpha=0$ and 0.35 respectively, estimated by the data in Fig. $13 \mathrm{I}$ and II. For $\beta \sim 1$ and regardless of the value of $\alpha, g(\tilde{H})$ has the double-peak structure on account of the delocalization of $\left\langle S_{i}^{z}\right\rangle$ with mild curvature. Indeed, such a structure has been observed in the 
NMR spectrum for $\mathrm{Sr}_{2} \mathrm{CuO}_{3}$ at a low temperature [see Fig. $2(30 \mathrm{~K})$ of ref. 78$]$. In this spectrum the interval between the peaks looks narrower than that in Fig. 19 I $(L=200)$. This is interpreted as follows. The amplitude of $\left\langle S_{i}^{z}\right\rangle^{\mathrm{s}}$ (and also $\left\langle S_{i}^{z}\right\rangle^{\mathrm{u}}$ ) becomes smaller and flatter with increasing $L$, as explained in $§ 5.2$. Thus, the interval becomes narrower with increasing $L$, as observed in Fig.20(a). Ultimately, they are combined into a single peak for $L \rightarrow \infty$. Experimentally, the chain length of $\mathrm{Sr}_{2} \mathrm{CuO}_{3}$ is estimated at $L=1800 \pm 500$, namely longer but finite.

When the dimerization increases, the double peaks

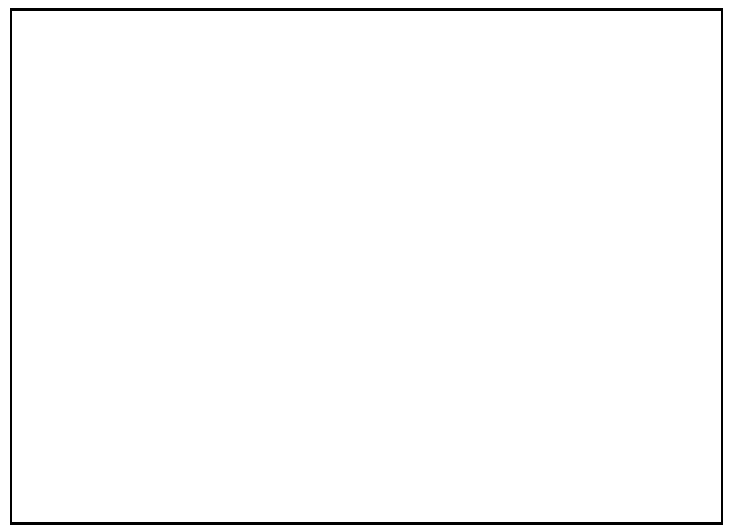

Fig. 20. System-size dependence of NMR intensity per spin (a) for the $S=1 / 2$ Heisenberg model, and for $\mathrm{CuGeO}_{3}$ (b) in the strong edge, and (c) in the weak edge. $\left\langle S_{i}^{z}\right\rangle^{\mathrm{s}}$ is used. The impurity density is proportional to $L^{-1}$. The width of the gaussian is $w=0.02$.

merge into a single peak, irrespective of the edge condition. This single peak is attributed to the disappearance of delocalized staggered moment, because the intensity of the peak at $\tilde{H} \sim 0$ originates from the dense distribution of $\left\langle S_{i}^{z}\right\rangle^{\mathrm{s}}$ near zero (see Fig. 13). There is one remarkable difference between the strong and weak edges; a broad skirt appears around the single peak for the latter, as seen in Fig. 19(b). This is caused by the localized free spins with large amplitude.

Finally, we refer to the influence of the impurity concentration. For $\beta=1$ the similar aspects to the Heisenberg case [see Fig. 20(a)] are observed for arbitrary values of $\alpha$. In Fig. 20(b) we show the system-size dependence of $g(\tilde{H})$ for the spin-Peierls phase of $\mathrm{CuGeO}_{3}$. With increasing $x(1 / L)$ the height of the single peak lowers and the spectrum becomes broad. Such impurity effect seems common to various systems.

\section{§. Haldane phase}

In this section, we study the edge effect in the Haldane phase, and clarify the difference from the spin liquid phase. As a typical compound, we refer to a F-AF bond-alternating compound monoclinic $\mathrm{CuNb}_{2} \mathrm{O}_{6}$. As discussed in Appendix D, this material is well described by the model eq. (1.1) with $\alpha=0$ and $\beta=-2.36(-2)$ for the pure (10\%-doped) sample. To avoid confusion, we make sure of the terms for edge condition: Corresponding to the strong (weak) edge in the spin-Peierls regime, we use terms "AF $(\mathrm{F})$ edge" for $\beta<0$, and " $J$ $(\beta J)$ edge" for a general value of $\beta$.

First of all, we consider the stability of the edge. In Fig. 21 we plot the energy difference $\Delta E$ in the ground states for a wide range of $\beta$ and $\alpha=0$. As mentioned for the spin-Peierls regime, the strong edge becomes stable, as soon as $\beta$ decreases from 1. However, as $\beta$ further decreases beyond $0, \Delta E$ starts to increase, and at last $E(\beta J)$ becomes lower than $E(J)$ at $\beta \sim-3$. For $\beta \rightarrow$ $\infty, \Delta E$ becomes $|\beta| J / 4=\infty$, because the number of $\mathrm{F}$ bonds is larger by one in the F-edge case. Thus, in the $S=1$ Haldane systems the F (weak) edge is always realized. Incidentally, the whole feature of $\Delta E$ including the reversal point -3 only weakly depends on the value of $\alpha$. In the bond-alternating systems with $|\beta| \ll \infty$ like monoclinic $\mathrm{CuNb}_{2} \mathrm{O}_{6}$, in which the lattice structure is rigid in contrast to the spin-Peierls compounds, two kinds of edge $J$ and $\beta J$ should be yielded at random by non-magnetic impurities.

Next, we discuss the spin polarization $\left\langle S_{i}^{z}\right\rangle$. As is

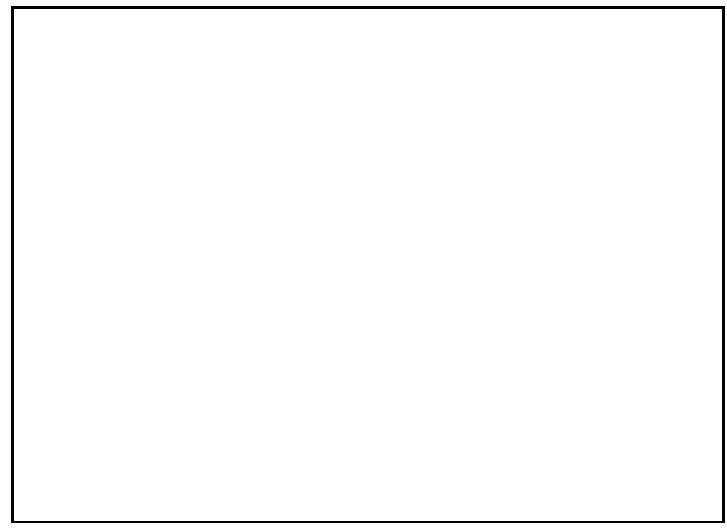

Fig. 21. Energy difference between the $J$ and $\beta J$ edges in the ground states. To show little size dependence, some systems of even $L$ are plotted together. $E(J)[E(\beta J)]$ indicates the total energy for the system of the $J(\beta J)$ edges. The minimum is $\Delta E / J=-0.75$ at $\beta=0$. The arrow indicates the reversal point at $\beta \sim-3$. This value 3 is roughly the ratio of energy gain in singlet $(3 J / 4)$ to triplet $(J / 4)$.

known for the $S=1$ Heisenberg model, ${ }^{33,68)}$ the magnetic excitation is localized at both edges as $S=1 / 2$ free spins. This localized moment decays exponentially with the localization length $\xi_{\text {edge }}$, which is independent of the system size and is equivalent to the bulk correlation length $\xi_{\mathrm{H}} \sim 6.03 .^{40,68)}$ Magnetic LRO's hardly appear even by doping, unless the interchain couplings are sufficiently strong. ${ }^{1,2,9)}$ Besides, localized edge spins have been actually observed by various experimental studies. $^{79)}$

We have already seen that such localization appears also in the present model, when the dimerization is introduced for the weak edge (Fig. 13). Then, we start with the $\mathrm{F}$ (weak) edge. In Fig. 22 we compare $\left\langle S_{i}^{z}\right\rangle$ 
Fig. 19. Intensity of NMR spectra $g(\tilde{H})$ for $\alpha=0$ (I) and 0.35 (II) for the segment of $L=200$. The strong- and weak-edge cases are shown in (a) and (b), respectively. In II(a) the line shapes are almost the same for $\beta=0.40-0.95$. They are obtained from the staggered part of the data in Fig. 13, since $\left\langle S_{i}^{z}\right\rangle^{\mathrm{u}}$ only weakly depends on $i$ for $\beta \sim 1$. The width of the gaussian is $w=0.02$.

in the Haldane phase $\left(\mathrm{CuNb}_{2} \mathrm{O}_{6}\right)$ with the spin liquid $\left(\mathrm{CuGeO}_{3}\right)$. For $\mathrm{CuNb}_{2} \mathrm{O}_{6}$ (open circle), $\left|\left\langle S_{i}^{z}\right\rangle\right|$ obeys typical exponential decay, in contrast to the spin-Peierls cases [see Fig. 13(b)]. Besides, $\xi_{\text {edge }}$ is fairly short and is almost the same with the bulk correlation length $\xi \sim 3.3$ (Fig. 5). By comparing Figs. 22(a) and (b), we find that the amplitude of $\left\langle S_{i}^{z}\right\rangle$ near the edge is independent of the system size. On the other hand, not only the oscillating part but also the uniform part of $\left\langle S_{i}^{z}\right\rangle$ vanishes for the bulk. Since the situation $\left\langle S_{i}^{z}\right\rangle=0$ is the same with the ground state $(S=0)$, the magnetic excitation never affects the bulk. Thus, the behaviors of the Haldane phase in the $S=1$ systems stays unchanged for the present case. Incidentally, the odd-site systems show similar behaviors to this case, as discussed in $§ 5.2$.

As for the AF (strong) edge, the excess spins extend to the whole system (small open diamond in Fig. 22). Nevertheless, the oscillating part is completely lacking. Thus, the magnetic excitations are not likely to induce LRO also in this case. Indeed, monoclinic $\mathrm{CuNb}_{2} \mathrm{O}_{6}$ keeps the gap properties in heavily doped samples, ${ }^{8)}$ in spite of the relatively large interchain coupling (cf. Appendix D). In contrast, for $\mathrm{CuGeO}_{3}$ the excited moment extends to the whole system (solid circle in Fig. 22). Moreover, the amplitude of staggered part abruptly grows with decreasing $L$ (increasing $x$ ). Thus, the magnetic excitation leads to AFLRO, as has been already discussed.

Let us turn to the NMR spectra. In Fig. 23 we show the size dependence of the NMR intensity per spin for the parameters of pure $\mathrm{CuNb}_{2} \mathrm{O}_{6}$. For a low impurity concentration (large $L$ ) $g(\tilde{H})$ has a sharp peak at $\tilde{H} \sim 0$ for every edge condition, corresponding to the absence of large oscillating part of $\left\langle S_{i}^{z}\right\rangle$ in the bulk. For the $\mathrm{F}$ edge there appear skirts and broad side peaks due to the localized spins at the edges. Thus, the localization survives even in the low-temperature limit, so that the NMR

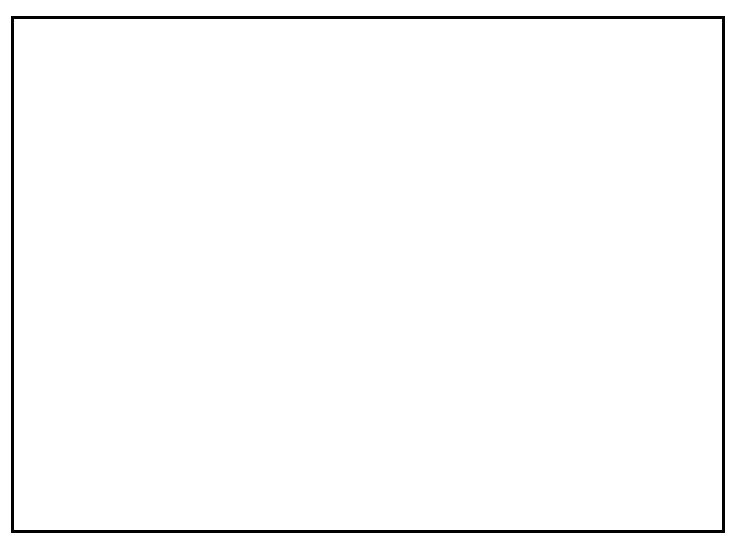

Fig. 22. Comparison of spin polarization in the lowest magnetic states between $\mathrm{CuGeO}_{3}$ (strong edge) and $\mathrm{CuNb}_{2} \mathrm{O}_{6}$ (two kinds of edges) for the systems of (a) $L=100$ and (b) 50. As for parameter values, $(\alpha, \beta)=(0.35,0.98)$ is assumed for $\mathrm{CuGeO}_{3}$, and $(0.0,-2.36)$ for $\mathrm{CuNb}_{2} \mathrm{O}_{6}$.

line shape will hardly broaden with decreasing temperature, unlike the $S=1 / 2$ Heisenberg compound ${ }^{78)}$ and the two-leg ladder system. ${ }^{6)}$ On the other hand, when the impurity concentration increases ( $L$ becomes small), the height of the single peak at $\tilde{H} \sim 0$ lowers regardless of the edge condition. Simultaneously, the peak shifts slightly for the AF edge, while the intensity of the skirts and side peaks grow for the F edge. Anyway, the Haldane phase and the spin liquid share the tendency toward the breakdown of the sharp peak by a few percent of impurity.

In conclusion, we recapitulate the relationship between the phase transition and the impurity effect. The characteristics of magnetic excitation in the Haldane phase are that the excited moment is confined in a finite range 


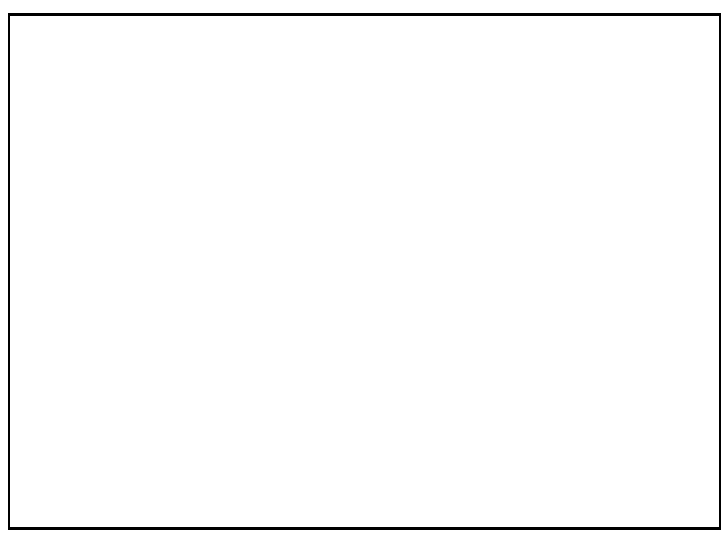

Fig. 23. System-size dependence of the NMR intensity per spin for $\mathrm{CuNb}_{2} \mathrm{O}_{6}$ (a) in the $\mathrm{AF}$ edges and (b) in the $\mathrm{F}$ edges. For odd-site systems, $g(\tilde{H})$ is analogous to (b) with the doubled system size. We use the fixed value of $\beta=-2.36$ (for pure material) for simplicity, although the value of $\beta$ changes with the impurity concentration (see Appendix D). To obtain the spectra by eq. (5.5), total $\left\langle S_{i}^{z}\right\rangle$ (including uniform part) is used with $w=0.02$.

near the $\beta J$ edges, and that the oscillating part in $\left\langle S_{i}^{z}\right\rangle$ is almost absent for the $J$ edges. The Haldane phase occupies the whole $\alpha-\beta$ plane except for the $\alpha$ axis and $\alpha=\infty$, on which the phase is the spin liquid characterized by the delocalization of $\left\langle S_{i}^{z}\right\rangle$. As the parameters approach the spin liquid, the range of localized edge spins extends toward the bulk (independent of $L$ ) [ $\beta J$ edge], and the amplitude of oscillation $(<1 / L)$ in $\left\langle S_{i}^{z}\right\rangle$ grows $[J$ edge]. In the thermodynamic limit (for the pure material), however, both the range of edge spins [ $\beta J$ edge] and the amplitude of staggered moment [ $J$ edge] are still negligible. On the other hand, when the chains are cut into finite segments (for the doped case), the range of edge spins may extend to the greater part of (or whole) segment $[\beta J$ edge], and the amplitude of staggered moment may becomes sufficiently large $[J$ edge], according to the balance between $\beta$ and $L(x)$. Thus, the interchain coupling, which is quantitatively important, has a chance to induce AFLRO. In the transition areas from the Haldane phase to the spin liquid, impurities play a dramatic role.

So far, we have not mentioned the two-leg ladder systems. In the typical ladder compounds, AFLRO is observed for the pure $\left.\left(\mathrm{LaCuO}_{2.5},{ }^{80}\right) T_{\mathrm{N}} \sim 110 \mathrm{~K}\right)$ and lightly-doped $\left(\mathrm{SrCu}_{2} \mathrm{O}_{3}{ }^{5,6)}\right)$ cases. At the early stage these compounds were considered as isotropic ladders $\left(J_{\text {leg }} / J_{\text {rung }}=\alpha=1\right)$, while recent experiments have concluded $\alpha \gtrsim 2 .{ }^{6,81)}$ Actually, as described in $\S 3$, the isotropic ladder point $[(\alpha, \beta)=(1,0)]$ exhibits a typical properties of the Haldane phase with $\xi \sim 3$ without the tendency toward AFLRO. However, our preliminary calculations of various quantities as well as a previous study $^{54)}$ indicate that the aspects for $\alpha \gtrsim 2$ are rather similar to the $S=1 / 2$ Heisenberg model. Thus, it is probable that the impurity effect of $\mathrm{SrCu}_{2} \mathrm{O}_{3}$ will be sim- ilarly understood by considering that the system is situated in the transition area from the Haldane phase to the spin liquid in the two-chain Heisenberg limit $(\alpha \rightarrow \infty)$. We will publish the results for ladder elsewhere.

\section{§. Summary}

We have studied a variety of properties of the 1D Heisenberg model with the bond alternation and the NNN exchange coupling, using the DMRG and ED methods. We summarize our main results below. As for the bulk properties before doping:

(1) The conditions for the Haldane phase are satisfied on the Shastry-Sutherland line $(\beta=2 \alpha)$ for $0 \leq \alpha<0.5$. A transition from the Haldane phase to a kind of spin liquid occurs at the Majumdar-Ghosh point $(\alpha=0.5)$.

(2) The above result can be extended to the whole $\alpha-\beta$ space. The Haldane phase continuously connects the $S=1$ limit $(\beta=-\infty)$ to the spin-Peierls regime $(\beta \sim 1)$ through the bond-alternating regime, and the transition occurs at $\beta=1$. In the Haldane phase, there is no anomaly in the dimer and string order parameters, whereas the spin gap and the correlation length exhibit singularities due to the incommensurate correlation.

(3) We have determined the phase diagram of the incommensurate correlation. The range of essential incommensurate correlation defined by $\left\langle S_{i}^{z} S_{j}^{z}\right\rangle$ is quite different from that by $S(q)$. The singular points of the spin gap are included in the incommensurate range of $S_{i j}$.

(4) We have discussed various properties, especially dynamics, of monoclinic $\mathrm{CuNb}_{2} \mathrm{O}_{6}$. This compound is well described by eq. (1.1) as a typical ferromagneticantiferromagnetic bond-alternating chain.

As for the edge effects, corresponding to the doping of non-magnetic impurities:

(5) There are two kinds of chain ends, according as the edge bond is strong $(J)$ or weak $(\beta J)$. The strong edge is more stable for the spin-Peierls regime, while the weak $(\mathrm{F})$ edge becomes stable for $\beta \lesssim-3$. This fact is possibly connected to the compositional phase transition of the first order between the dimerized and uniform $\mathrm{AF}$ phases in $\mathrm{CuGeO}_{3}$.

(6) The spin polarization is delocalized in the spinPeierls systems and the two-leg ladders with large $J_{\text {leg }} / J_{\text {rung }}$, while the spin polarization is localized at the edge for the bond-alternating systems as well as the Haldane systems and the isolated dimers. In the former, $\left\langle S_{i}^{z}\right\rangle$ is enhanced by the impurity and triggers a 3D magnetic long-range order. In the latter, $\left\langle S_{i}^{z}\right\rangle$ is insensitive to the impurity, so that the gap properties are preserved.

(7) For finite but large systems NMR spectra in the low temperatures show a double-peak structure in the vicinity of $\beta=1$ due to the delocalized spin polarization. On the other hand, a single sharp peak appears for the Haldane phase, regardless of the edge condition. In this phase, the conspicuous broadening of the spectra with decreasing temperature will not be observed. Generally, as the impurity concentration is increased, the height of peak lowers and eventually vanishes. 


\section{Acknowledgments}

We thank Masatoshi Sato and his group for providing us of experimental data on $\mathrm{CuNb}_{2} \mathrm{O}_{6}$ prior to publication as well as useful discussions. We are grateful to Takashi Yamamoto, Kazuma Hirota and Zenji Hiroi for valuable discussions, and to Tota Nakamura for informing us of some references. A part of the numerical calculations was carried out with VPP500 at the Supercomputer Center of ISSP, University of Tokyo. Parallelized fortran code was executed by SX4R at the Supercomputer Center, Tohoku University and CP-PACS computer of Center for Computational Physics, University of Tsukuba. This study is partly supported by Grant-in-Aids for Encouragement of Young Scientists given by the Ministry of Education, Science, Sports and Culture.

\section{Appendix A: Relations between two represen- tations}

In this Appendix, we summarize the relations between the Hamiltonians eq. (1.1) and eq. (2.3). Comparing corresponding terms, one obtains the relations,

$$
\delta=\frac{1-\beta}{1+\beta}, \quad \tilde{\alpha}=\frac{2 \alpha}{1+\beta}, \quad \tilde{J}=\frac{1+\beta}{2} J .
$$

Thereby, a line (point) in the $\alpha-\beta$ plane is moved to another line (point) in the $\tilde{\alpha}-\delta$ plane. Note that the Shastry-Sutherland line is $\beta=2 \alpha$ in eq. (1.1) and $2 \tilde{\alpha}+\delta=1$ in eq. (2.3). The isotropic ladder point is $(\alpha, \beta)=(1,0)$ or $(\tilde{\alpha}, \delta)=(2,1)$. However, the $\alpha$ axis $(\alpha, \beta=1)$ remains the $\tilde{\alpha}$ axis $(\tilde{\alpha}, \delta=0)$. The energy scale also should be changed; $E$ in eq. (1.1) is related to $\tilde{E}$ in eq. (2.3) as,

$$
\frac{\tilde{E}}{\tilde{J}}=\frac{2}{1+\beta} \frac{E}{J} .
$$

\section{Appendix B: Cusp Behavior of the Gap}

In this Appendix we pursue the cusp behavior in $\Delta$ to supplement the discussions in $\S 3.1$.

The second-order perturbation energies for the ground state $(S=0)$ and the lowest excited branches $(S=1)$ around the isolated dimer point are given by eqs. (3.1) and (3.2), respectively. The singlet energy is lowered by the second-order perturbation for $\beta \neq 2 \alpha$; but this decrement vanishes for $\beta=2 \alpha$ (the SS line). This cancellation is understood intuitively as follows: Two $\alpha$ bonds and one $\beta$ bond connect two singlet dimers, but $\alpha$ and $\beta$ play mutually inverse roles for the spin directions in the two singlet pairs. On the other hand, the triplet branch has a dispersion due to the motion in the array of singlets for $\beta \neq 2 \alpha$. The bottom of $E_{t}(q)$ is located at $q=0$ for $\beta>2 \alpha$ and at $q=\pi / 2$ for $\beta<2 \alpha$; the IC value of $q$ does not appear around the isolated dimer point. Thus,
$\Delta$ is obtained as:

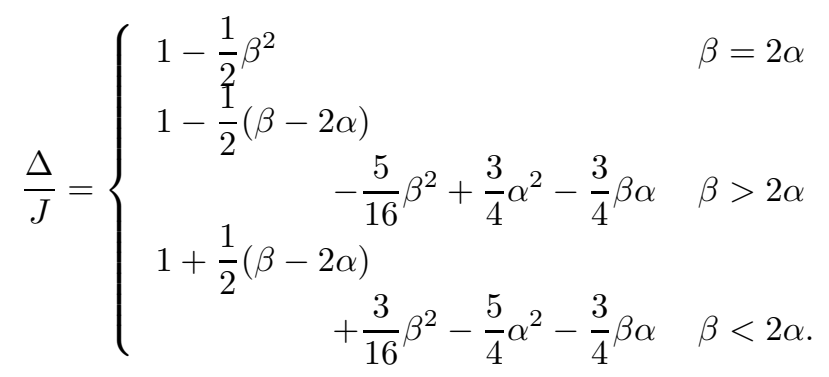

Comparing the first-order terms, one finds the maximum of $\Delta$ is at $\beta=2 \alpha$. The cusp behaviors including the curvature in both sides of $\beta=2 \alpha$ are reproduced by the differential coefficients:

$$
\left\{\begin{array}{lll}
\frac{\partial \Delta}{\partial \beta}<0, & \frac{\partial^{2} \Delta}{\partial \beta^{2}}<0 & \beta>2 \alpha \\
\frac{\partial \Delta}{\partial \beta}>0, & \frac{\partial^{2} \Delta}{\partial \beta^{2}}>0 & \beta<2 \alpha .
\end{array}\right.
$$

With increasing $\alpha$, however, the cusp point deviates to the side of $\beta<2 \alpha$ as seen in Fig 12 .

Then, let us look at the cases of finite $\alpha$ with the ED results. In Fig. 24(a) the lowest triplet excitation for the available momenta in $L=24$ are plotted for $\alpha=$ 0.35. The boundary between the lowest excitations of $q=\pi$ and $q=\pi / 2$ still forms the cusp near $\beta=0.525$. An interesting point is that the excitation energies for all the momenta meet at the cusp just like the isolated dimers. No dispersion in the lowest excitation is evident in $S(q, \omega)$ (Fig. 25); the excitation has a local character at the cusp points.

The above feature continues to $\alpha \sim 0.5$, beyond which the lowest excitation is no longer restricted to the $\mathrm{C}$ wave numbers. As in Fig. 24(b) for $\alpha=0.6$, the wave number of the lowest excitation does not switch directly from $q=\pi / 2$ to $\pi$, but IC excitations lie between them. Thus, the cusp behavior disappears for $\alpha \gtrsim 0.5$, as seen for $\alpha=1$ in Fig. 3.

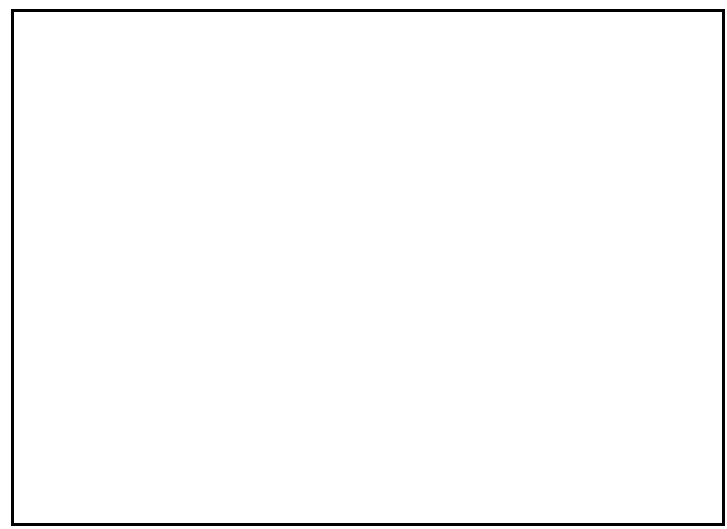

Fig. 24. Excitation energy of the lowest triplet branches for each available value of $q$ for $L=24$; (a) $\alpha=0.35$ and (b) 0.6. The data are obtained by ED and the recursion method. 


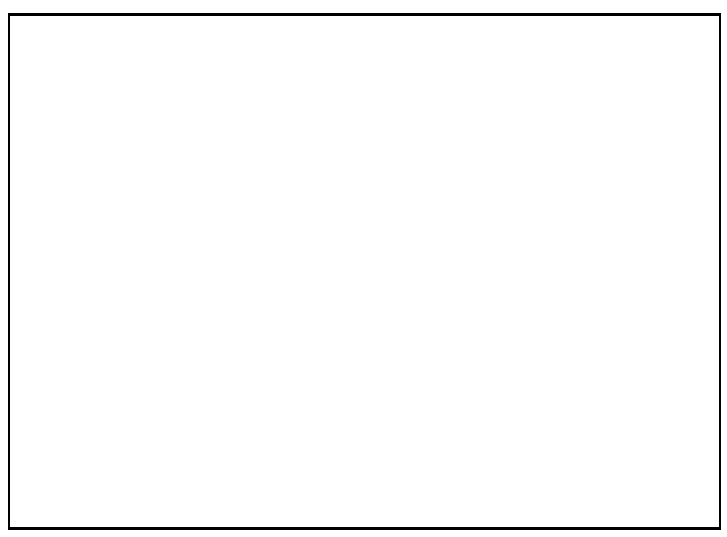

Fig. 25. Dynamical structure factor $S(q, \omega)$ for a cusp point $(\alpha, \beta)=(0.35,0.525)$. Intensity is proportional to the area of circle. Data for $L=14-26$ are plotted together. Note that every branch in this figure is independent of $L$.

\section{Appendix C: Low-Energy States on the Shastry- Sutherland Line}

The isolated dimer point satisfies the conditions of the Haldane phase itemized in $\S 2$, while the MG point breaks the condition of a unique ground state. Hence, the phase is different between these points, which are the two ends of the SS line. With a phase transition in mind, we summarize and reconsider the knowledge of the ground state and low-lying excitations along the SS line in this Appendix. In $\S$ C.1 using a non-local unitary transformation, we explain that the SS line strictly satisfies the conditions of the Haldane phase. In $\S \mathrm{C} .2$ we summarize the structure of energy levels under different boundary conditions with the ED data. In $\S$ C.3 we show that the transition occurs just at the $\mathrm{MG}$ point in terms of the perturbation theory for the degenerate case.

\section{C.1 Non-local unitary transformation}

Following the formulation of the non-local unitary transformation by Takada and Kubo, ${ }^{29,82)}$ we rewrite the Hamiltonian eq. (1.1) as

$$
\begin{aligned}
& \mathcal{H} / J=-\sum_{i=1}^{L-1}\left(\sigma_{i}^{x} \sigma_{i+1}^{x}+\tau_{i}^{z} \tau_{i+1}^{z}+4 \sigma_{i}^{x} \tau_{i}^{z} \sigma_{i+1}^{x} \tau_{i+1}^{z}\right) \\
&-\alpha \sum_{i=1}^{L-1}\left[\sigma_{i}^{x} \tau_{i+1}^{x}+\sigma_{i}^{z} \tau_{i+1}^{z}+\tau_{i}^{x} \sigma_{i+1}^{x}+\tau_{i}^{z} \sigma_{i+1}^{z}\right. \\
&\left.+4\left(\sigma_{i}^{x} \tau_{i}^{z} \sigma_{i+1}^{z} \tau_{i+1}^{x}+\tau_{i}^{x} \sigma_{i}^{z} \tau_{i+1}^{z} \sigma_{i+1}^{x}\right)\right] \\
&+\beta \sum_{i=1}^{L} \boldsymbol{\sigma}_{i} \cdot \boldsymbol{\tau}_{i},
\end{aligned}
$$

where the operator $\boldsymbol{S}$ is replaced by $\boldsymbol{\sigma}_{i}=\boldsymbol{S}_{2 i}$ and $\boldsymbol{\tau}_{i}=$ $\boldsymbol{S}_{2 i-1}$. As for the isolated dimer point $(\alpha=\beta=0)$, the surviving part [the first line of eq. (C.1)] is coupled Ising chains with a $Z_{2} \times Z_{2}$ symmetry. The ground state has ferromagnetic orders in both $\sigma_{i}^{x}$ and $\tau_{i}^{z}$, and is fourfold degenerate $\left(\sigma_{i}^{x}= \pm 1 / 2\right.$ and $\left.\tau_{i}^{z}= \pm 1 / 2\right)$; the symmetry is completely broken. ${ }^{28,29)}$ Moreover, since the eigenstate is $\psi_{1}$ of eq. (2.1) (or $\psi_{2}$ ), there exists a single-triplet gap of magnitude $J$. The string order parameter is calculated as $1 / 4$. Thus, the conditions of the Haldane phase are satisfied at this point. These conditions are still satisfied on the SS line for $0 \leq \alpha<0.5$, because the ground state is identical with that of the isolated dimer point, and is unique with a finite singlet-triplet gap. We will make sure of the latter point below. This result has been also reached with the matrix product procedure. ${ }^{17}$ )

\section{C.2 Structure of low-energy levels}

Here, we study the low-energy levels on the SS line $(\delta+2 \tilde{\alpha}=1)$ for finite systems. The boundary condition greatly affects the level structure.

Figure 26(a) shows the ED result under $\mathrm{PBC}$ in the $S^{z}=0$ sector. Under this boundary condition there is no degeneracy in the ground state in accordance with the conditions of the Haldane phase. At the isolated dimer point, the first excited states are triplet and $3 L / 2$-fold degenerate. Although this degeneracy is lifted by a finite value of $\tilde{\alpha}$, the energy difference is very small except for the vicinity of the MG point. There, as seen in the inset, the level of $S=0$, which is one of the degenerate ground states at the MG point, goes up linearly with decreasing $\tilde{\alpha}$, and intersects the lowest $S=1$ level at $\tilde{\alpha} \sim 0.48$ (indicated by arrow). This means that the lowest excited state becomes singlet near the MG point. However, the range of this singlet-singlet excitation depends on the system size. In Fig. 27 we show similar figures with different system sizes. With increasing $L$, the crossing point approaches the MG point. This point will be pursued in $\S$ C.3.

In Fig. 26(b) plotted is the result for OBC with the strong edges. In this case the ground state is either $\psi_{1}$ or $\psi_{2}$ according as singlet pairs sit on the edge bonds, and always nondegenerate. Note that the edge bonds are occupied by singlet pairs even for the non-dimerized (MG) case. The first excited state is always triplet. Thus, not only the ground state is identical with that of $\mathrm{PBC}$, but properties of excitation are also analogous to those of PBC. We have taken advantage of such a property to obtain the bulk gap in $\S 3.1$.

Figure 26(c) shows the same for OBC with the weak edges. In this case, there is the pseudo fourfold (twofold in the $S^{z}=0$ sector) degeneracy except for the vicinity of the MG point. The ground state $(S=0)$ is neither $\psi_{1}$ nor $\psi_{2}$ except for the MG point, due to the isolated spins at the edges. Thus, this edge condition has a feature specific to the open edge of the Haldane systems. The nearly degenerate lowest levels ( $S=0$ and $S=1$ ) separate at $\tilde{\alpha} \sim 0.46$; the $S=0$ level abruptly comes down and converges to the value of $\psi_{1}$ (or $\psi_{2}$ ) at the MG point. In this process the singlet array is rearranged, say from $L$ to $R$, to adapt itself to the strong edges. On the other hand, near the above separation point the second lowest $S=0$ level, which has a similar energy to the lowest $S=1$ level near the MG point, abruptly changes its direction and merges into the upper $S=1$ levels. These behaviors correspond to the level crossing under PBC mentioned above, and the separation point also highly depends on 


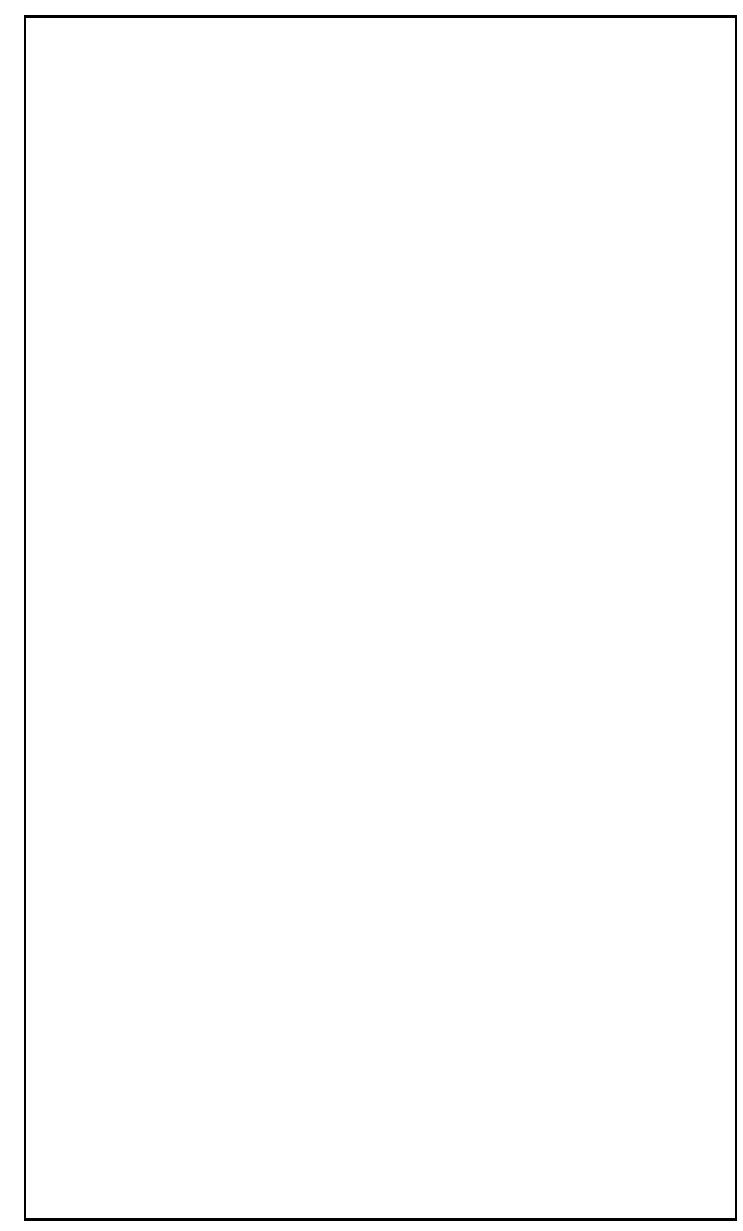

Fig. 26. Some lowest levels in the $S^{z}=0$ subspace on the SS line $(\delta+2 \tilde{\alpha}=1)$ as a function of $\tilde{\alpha}$; (a) $\mathrm{PBC}(L=16)$, (b) OBC with the strong edges $(L=12)$, and (c) OBC with the weak edges $(L=12)$. The insets are respective magnifications near the MG point. The arrow in the inset of (a) indicates the crossing point of the second lowest $S=0$ level and the lowest $S=1$ level

Fig. 27. Three lowest levels on the SS line $(\delta+2 \tilde{\alpha}=1)$ near the MG point in the $S^{z}=0$ sector under PBC. The system size is different between (a) and (b) [see also the inset of Fig. 26 (a)]. The Arrow indicates the crossing point of the lowest $S=1$ level and the second lowest $S=0$ level. the system size. This point will be taken up again in $\S$ C.3.

In this connection, recent studies ${ }^{83)}$ have discussed the appearance of the mid-gap states for the doped $\mathrm{CuGeO}_{3}$ at $T_{\mathrm{N}}<T<T_{\mathrm{SP}}$. According to the above results, however, mid-gap levels are not likely to arise from the strong edges, the case for $\mathrm{CuGeO}_{3}$. On the other hand, for the weak edges (including odd-site systems) the energy difference among the nearly fourfold degenerate states increases exponentially with $1 / L$. Thus, the experiment may detect such levels, if any.

Finally, we generalize the level structure for the bondalternating systems, aside from the behaviors near the MG point. Under PBC or OBC with the strong edges, the ground state is a nondegenerate singlet $\left(\psi_{1}\right.$ or $\psi_{2}$ in the case of the SS line) with the singlet-triplet gap over it. Under OBC with the weak edges, the ground state is nearly fourfold degenerate (one singlet and three triplets) with the bulk gap over these four states.

\section{C.3 Breakdown of the perturbation theory}

Having seen extraordinary behaviors around the MG point in $\S$ C.2, here we reconsider them in the light of the perturbation theory for the degenerate case, and shows that the non-Haldane phase is restricted to the MG point.

The Hamiltonian eq. (2.3) on the SS line is written around the MG point as,

$$
\begin{gathered}
\mathcal{H}=\mathcal{H}_{\mathrm{MG}}+\zeta \mathcal{H}^{\prime}, \\
\mathcal{H}_{\mathrm{MG}}=\tilde{J} \sum_{i=1}^{L}\left[\boldsymbol{S}_{i} \cdot \boldsymbol{S}_{i+1}+\frac{1}{2} \boldsymbol{S}_{i} \cdot \boldsymbol{S}_{i+2}\right], \\
\mathcal{H}^{\prime}=-2 \tilde{J} \sum_{i=1}^{L}\left[(-1)^{i} \boldsymbol{S}_{i} \cdot \boldsymbol{S}_{i+1}+\frac{1}{2} \boldsymbol{S}_{i} \cdot \boldsymbol{S}_{i+1}\right],
\end{gathered}
$$

where we assume PBC and $\zeta=1 / 2-\tilde{\alpha}$ to be infinitesimal. Here, $\mathcal{H}_{\mathrm{MG}} \psi_{n}=E_{\mathrm{MG}} \psi_{n}(n=1,2)$ holds, where $E_{\mathrm{MG}}=-3 L \tilde{J} / 8$ is the ground-state energy at the $\mathrm{MG}$ point, and $\psi_{n}$ is assumed to be normalized. Let $\psi_{1}$ be the ground state on the SS line: $\mathcal{H}^{\prime} \psi_{1}=\varepsilon_{1} \psi_{1}$ with $\varepsilon_{1}=-3 L \tilde{J} / 4{ }^{24)}$ Since $\psi_{1}$ and $\psi_{2}$ are orthogonal in the thermodynamic limit and $\psi_{1}$ is still an eigenstate under the perturbed Hamiltonian, the first-order perturbation energies for the two levels are formally written in a simple form, as

$$
E_{n}^{\prime}=\zeta\left\langle\psi_{n}\left|\mathcal{H}^{\prime}\right| \psi_{n}\right\rangle=\zeta \varepsilon_{n} \quad(n=1,2) .
$$

Thus, the perturbation theory naturally works for the lower level $\psi_{1}^{\prime}=\psi_{1}$. As for the higher level $\psi_{2}^{\prime}$, if the perturbation theory works, the energy is also proportional to $1 / 2-\tilde{\alpha}$ near the MG point, actually as in the inset of Fig. 26(a) and in Fig.27. Since a direct calculation of $\varepsilon_{2}$ looks difficult, we estimate it by ED with PBC through,

$$
\varepsilon_{2}=\left\langle\psi_{2}\left|\mathcal{H}^{\prime}\right| \psi_{2}\right\rangle=\left.\frac{\partial E_{2}}{\partial \zeta}\right|_{\zeta \rightarrow 0},
$$

where $E_{2}$ is the total energy with respect to $\psi_{2}^{\prime}$. 
In Fig. 28(a) we plot $\tilde{J} / \varepsilon_{2}$ thus obtained versus $1 / L$. It shows that $\varepsilon_{2}$ diverges in the thermodynamic limit, namely the perturbation breaks down for $\psi_{2}^{\prime}$. Along with this phenomenon, the crossing point of $\psi_{2}^{\prime}$ and the lowest $S=1$ level converges to the MG point [Fig. 28(b)]. Thus, one of the degenerate levels at the $\mathrm{MG}$ point never continues to the dimerized region $(\delta>0)$; the first excited state under $\mathrm{PBC}$ is always triplet on the SS line.

On the other hand, as pointed out for OBC with weak edge in $\S$ C.2, the lowest level, which separates from the triplet levels near the MG point, behaves similarly with $\psi_{2}^{\prime}$ in PBC. We also estimate the perturbation energy $\varepsilon$ for this case in the same manner, and plot $\tilde{J} / \varepsilon$ together in Fig. 28(a). Within the numerical accuracy, the values of $\tilde{J} / \varepsilon_{2}$ and $\tilde{J} / \varepsilon$ are identical. Thus, the lowest level $(S=0)$ at the MG point does not smoothly continue to the $S=0$ state which makes up the fourfold degeneracy for $\delta>0$. And the degeneracy of the ground state continues to the limit $\tilde{\alpha} \rightarrow 1 / 2$. Thus, the energy difference between the strong and the weak edges in the limit of $L \rightarrow \infty$ and $\tilde{\alpha} \rightarrow 1 / 2$ becomes the MG gap.

From the above we conclude that the Haldane phase

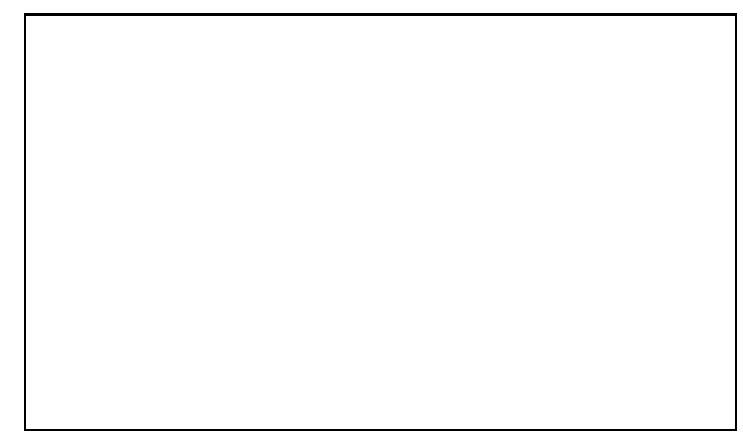

Fig. 28. (a) System-size dependence of the inverse of the firstorder perturbation energy per site for $\psi_{2}^{\prime}$ in PBC. The corresponding value for the lowest level in $\mathrm{OBC}$ with the weak edges are also shown. (b) System-size dependence of the crossing point of $\psi_{2}^{\prime}$ and the lowest $S=1$ level in PBC.

continues to this limit, and that the MG point is critical. Hence, the consequence of $\S \mathrm{C} .1$ is valid for $\delta \neq 0$. On the other hand, we can say inversely that in finite systems the properties of the Haldane phase are lost near the MG point. So far, we have restricted our discussion to the SS line. As far as we have tried numerically, however, this discussion is applicable to any path which starts from an arbitrary point on the $\alpha$ axis $\left(\alpha>\alpha_{\mathrm{c}}\right)$ and advances in an arbitrary direction of finite $\delta$.

\section{Appendix D: Bond-alternating compound $\mathrm{CuNb}_{2} \mathrm{O}_{6}$}

In this Appendix we discuss various aspects of a F-AF bond-alternating chain monoclinic $\mathrm{CuNb}_{2} \mathrm{O}_{6}$ to supplement the background of $\S 6$. The dynamical properties of bond-alternating chain, in particular for $\beta<0$, are surveyed in $\S$ D.1. In $\S \mathrm{D} .2$ various properties of $\mathrm{CuNb}_{2} \mathrm{O}_{6}$ are summarized, before discussing its dynamical proper- ties in $\S \mathrm{D} .3$.

\section{D.1 Spin dynamics for $\alpha=0$}

Since principal static properties have been already described in this paper as well as in some studies, ${ }^{26,29,84}$ ) we pay attention to the dynamics. As discussed in $§ 5.1$, low-lying spin excitation change its character from evennumber spinons to magnons, as soon as the bond alternation is introduced. Consequently, isolated magnon branches appear below the continuum. As in Figs. 29(a) and (b), the ratio of intensity (residue) in the lowest isolated branch increases with decreasing $\beta$ from 1 . At the isolated dimer point this branch has the total intensity with a completely flat band at $\omega=J$. For $\beta>0$ the wave number of gap is $\pi$ and the band top is at $\pi / 2$.

On the other hand, as $\beta$ decreases further for $\beta<0$, the main branch comes to have dispersion with a minimum at $q=\pi / 2$, as discussed by the perturbation calculations in Appendix B. A continuum due to two triplet pairs appears around $\omega=2 J$, as pointed out for $\beta \sim 0$ by Hida. ${ }^{26)}$ Furthermore, another isolated branch appears approximately at $\omega=|\beta| J$, which corresponds to the energy to break one $\mathrm{F}$ bond. In addition, higher harmonics appear, e.g. at $\omega \sim 6.5 J$ in Fig. 29 (d).

To begin with, let us look at the dominant lowestenergy branch (LEB) and the continuum. As shown in Fig. 10, the maximum of integrated intensity $S(q)$ moves from $q=\pi$ to $\pi / 2$ through IC wave numbers, as $\beta(<0)$ decreases. According to this change, and because the greater part of intensity is concentrated on LEB, the weight in LEB moves from $q=\pi$ to $\pi / 2$. For $\beta \lesssim-3$ the intensity at $q \sim \pi / 2$ is the strongest, and grows further with decreasing $\beta(<0)$ [Fig.30(a)]. Similarly, the weight of the continuum chiefly distributed at $q \sim \pi$ for small values of $|\beta|$ gradually moves to $q \sim \pi / 2$. We have found for $\beta \lesssim-2$ that the residue of LEB for $0.2<q / \pi<0.5$ is more than $85 \%$ and scarcely depend on the system size, whereas for $q \lesssim 0.2 \pi$ it considerably decreases with increasing $L$. This implies that LEB enters the continuum at $q \sim 0.2 \pi$. A similar aspect was observed for the $S=1$ Heisenberg model. ${ }^{85}$ )

Next, we mention the high-energy branch (HEB) near $\omega \sim|\beta| J$. The dispersion of HEB is flatter with a minimum also at $q / 2$. Accordingly, local destruction of a ferromagnetic bond chiefly contributes to this excitation. There exist weak continuum spectra around HEB, although HEB itself is an isolated branch because of little dependence of the intensity on $L$. As shown in Fig. 30(a) the intensity of HEB increases with decreasing $\beta$ at first; the residue for HEB at $q=\pi$ becomes as large as $70 \%$ for $\beta<-4$ in spite of the high-energy process. With decreasing $\beta(<0)$ further, however, its intensity decreases and vanishes in the Haldane limit, according to the total intensity $S(\pi)$ (Fig. 10). Therefore, this branch is specific to the F-AF bond-alternating systems.

\section{D.2 Monoclinic $\mathrm{CuNb}_{2} \mathrm{O}_{6}$}

As a spin-gap compound $\mathrm{CuNb}_{2} \mathrm{O}_{6}$ has been actively studied by various experimental means recently. ${ }^{8,15,86-88)} \quad \mathrm{CuNb}_{2} \mathrm{O}_{6}$ has zigzag chains of edgesharing $\mathrm{CuO}_{6}$ octahedra with localized $S=1 / 2$ spins 
Fig. 29. Dynamical structure factor $S(q, \omega)$ for four values of $\beta(\delta)$ and $\alpha=0$. Data obtained by the ED with a recursion method for $L=14-26$ are simultaneously plotted. The intensity is proportional to the area of circle. In (a) and (b), to convert the $\tilde{\alpha}-\delta$ representation into the $\alpha-\beta$ representation, $5 / 6$ and $2 / 3$ have to be multiplied to $\omega$, respectively. See Appendix A.

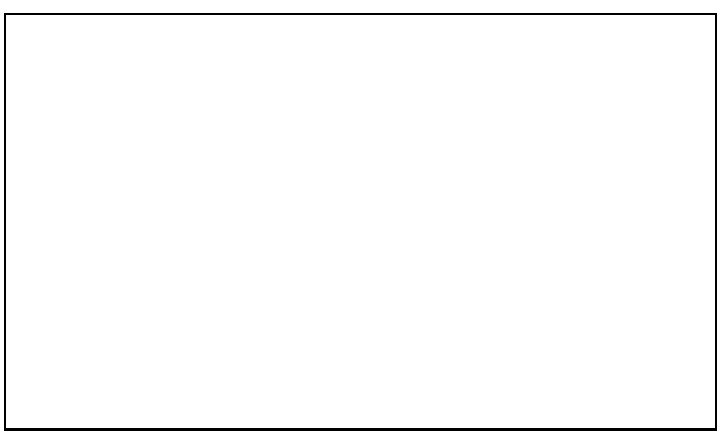

Fig. 30. (a) Intensity $[S(q, \omega)]$ of LEB at $q=\pi / 2$ (solid circle) and of HEB at $q=\pi$ (open circle) as a function of $-\beta$. The data points are extrapolated from the ED results of $L=14-26$. (b) Excitation energy of LEB at $q=\pi / 2\left[\omega_{\mathrm{L}}(=\Delta)\right]$ and of HEB at $q=\pi\left[\omega_{\mathrm{H}}\right]$, and their ratio are shown as a function of $-\beta$. These values are estimated by the DMRG and ED methods $(L \rightarrow \infty)$ for eq. (1.1) with $\alpha=0$. The experimental value of the ratio for $10 \%$-doped $\mathrm{CuNb}_{2} \mathrm{O}_{6}$ are indicated by the dashed line.

on $\mathrm{Cu}^{2+}$ ions. This compound has two polymorphs, i.e. orthorhombic and monoclinic phases. The orthorhombic phase is considered as a 1D Heisenberg antiferromagnet with an AFLRO below 7.3K; on the other hand, the monoclinic phase shows thermal-activation-type behaviors in magnetic susceptibility $\chi(T) .{ }^{86)}$ Henceforth, we restrict our discussions to the monoclinic phase. $\mathrm{Ow}-$ ing to the zigzag structure and the neutron scattering data of the lowest excitation at $q=\pi / 2$, it is expected that monoclinic $\mathrm{CuNb}_{2} \mathrm{O}_{6}$ should be a $\mathrm{F}-\mathrm{AF}$ bond-alternating chain. Actually, the experimental $\chi(T)$ was fitted successfully to the results of the model eq. (1.1) with $J=51 \mathrm{~K}, \alpha=0$ and $\beta=-2.36 .^{8)}$ Simultaneously, the coincidence in specific heat $C(T)$ is satisfactory with the same parameter set. Our estimation $\Delta / J=0.3714$ for these values (Fig. 3) leads to $\Delta=19 \mathrm{~K}$.

Notwithstanding, recalling the ambiguity in modeling a spin-gap systems based solely upon $\chi(T)$, for example $(\mathrm{VO})_{2} \mathrm{P}_{2} \mathrm{O}_{7}{ }^{89)}$ and $\mathrm{KCuCl}_{3},{ }^{90)}$ one should run a careful check by other means, in particular neutron scattering.

Before discussing the neutron experiment, we touch on a couple of related properties of $\mathrm{CuNb}_{2} \mathrm{O}_{6}$. First, we consider the effect of the NNN coupling. Fitting the experimental $\chi(T)$ to the ED results of $L=16^{91)}$ around $(\alpha, \beta)=(0,-2.36)$, we have found that a series of parameter sets such as $(0.10,-2.0),(0.24,-1.5)$ and $(0.38,-1.0)$ bring favorable results in the same degree with $(0,-2.36)$. This reminds us of the tendency that $\alpha$ encourages the $\uparrow \uparrow \downarrow \downarrow$ correlation. Concerning $C(T)$, however, such a set leads to a poorer fit, as $\alpha$ increases. Hence, we conclude that the NNN exchange is negligibly small in $\mathrm{CuNb}_{2} \mathrm{O}_{6}$ despite the edge-sharing linkage. This is intuitively understood by considering the difference in the orbital structure between $\mathrm{CuGeO}_{3}$ and $\mathrm{CuNb}_{2} \mathrm{O}_{6}$. In $\mathrm{CuGeO}_{3}$ the NNN exchange arises from a superexchange process through the path of $\mathrm{Cu}-\mathrm{O}-\mathrm{O}-\mathrm{Cu}$ in the $\mathrm{CuO}_{2}$ plane. ${ }^{11)}$ In this case a hybridized $\mathrm{Cu}-\mathrm{O}$ orbital overlaps the counter $\mathrm{Cu}-\mathrm{O}$ orbital to some extent. On the other hand, in $\mathrm{CuNb}_{2} \mathrm{O}_{6}$ the two $\mathrm{Cu}-\mathrm{O}$ hybridized orbitals of our concern are not in a plane and do not overlap each other because of the zigzag linkage of $\mathrm{CuO}_{6}$ octahedra. This comparison affords vital support to the above mechanism of the NNN exchange in the edge-sharing compounds.

Next, we consider the effect of elemental substitu- 
tion. ${ }^{8)}$ In the crystal of monoclinic $\mathrm{CuNb}_{2} \mathrm{O}_{6}, \mathrm{Cu}$ can be replaced by $\mathrm{Zn}$ up to $40 \%$. In sharp contrast to $\mathrm{CuGeO}_{3}$, the gap behaviors remain and no magnetic LRO appears even for the 40\%-doped sample. In doped samples, however, lattice constants and bond angles change according to the $\mathrm{Zn}$ concentration, mainly due to the different atomic radii between $\mathrm{Cu}$ and $\mathrm{Zn}$; this change, particularly in the bond angles, ought to affect the exchange integral, according to the Kanamori-Goodenough rule. ${ }^{92)}$ Actually, considerable changes in parameters occur as summarized in Table II, ${ }^{93)}$ where the values are estimated by the fit of $\chi(T)$ for some doped samples $\mathrm{Cu}_{1-x} \mathrm{Zn}_{x} \mathrm{Nb}_{2} \mathrm{O}_{6}$. Also entered in Table II is the gap estimated through eq. (1.1) using those parameters. The decreasing tendency versus $x$ coincides with the gap estimated from $C(T)$, which decreases linearly with $x .^{8}$ ) This behavior is contrary to what the $S=1$ Haldane systems show; the gap increases with increasing $x .{ }^{94)}$ The reason is that thick doping cuts the chains into shorter segments, and that the gap generally increases as the system size becomes small. In the case of $\mathrm{CuNb}_{2} \mathrm{O}_{6}$ the decrease of $|J|$, which is specific to this compound, probably overcomes the system-size dependence of the gap and the increase of $\beta$.

Table II. Parameters for the doped $\mathrm{CuNb}_{2} \mathrm{O}_{6}$ obtained from the fit of $\chi(T)$. The gap $\Delta$ is estimated through the model eq. (1.1).

\begin{tabular}{cccc}
\hline$x$ & $J(\mathrm{~K})$ & $\beta$ & $\Delta(\mathrm{K})$ \\
\hline 0.0 & 51.4 & -2.36 & 19.1 \\
0.1 & 44.5 & -1.95 & 18.9 \\
0.2 & 40.0 & -1.86 & 17.6 \\
0.3 & 33.4 & -1.43 & 17.2 \\
0.4 & 28.1 & -1.03 & 17.1 \\
\hline
\end{tabular}

\section{D.3 Inelastic neutron scattering}

Recently an inelastic neutron scattering experiment was performed on an $\mathrm{Zn}$-doped $\mathrm{CuNb}_{2} \mathrm{O}_{6}$ sample. ${ }^{15)}$ The spectra obtained in this experiment have reproduced the most features of the F-AF bond-alternating chain discussed in $\S$ D.1. We owe all the experimental data in this subsection to ref. 15.

We begin with the evaluation of the parameters. In Fig. 30(b) shown are the excitation energies of LEB $\omega_{\mathrm{L}}$ at $q=\pi / 2\left(\omega_{\mathrm{L}}=\Delta\right)$, and of HEB $\omega_{\mathrm{H}}$ at $q=\pi$, and their ratio $\omega_{\mathrm{H}} / \omega_{\mathrm{L}}$, estimated by the DMRG and ED methods for eq. (1.1) with $\alpha=0$. On the other hand, the experimental values are $\omega_{\mathrm{L}}^{\mathrm{ex}}=1.73 \mathrm{meV}$ and $\omega_{\mathrm{H}}^{\mathrm{ex}}=13.14 \mathrm{meV}$, respectively. Then, the ratio becomes $\omega_{\mathrm{H}}^{\mathrm{ex}} / \omega_{\mathrm{L}}^{\mathrm{ex}}=7.6$, which is shown with dashed line in Fig. 30(b). Thus, we find that the value $\beta$ for $\mathrm{CuNb}_{2} \mathrm{O}_{6}$ of $10 \%$ substitution is about -2.0 , which agrees well with the values estimated by $\chi(T)$ in Table II.

In Figs. 31(a) and (b), we depict the excitation energy of LEB and of HEB, respectively, for the whole $q$ space. In these figures we also plot the experimental results with $J=46.9 \mathrm{~K}$, which best fits the whole data (both for LEB and HEB) at the same time to the calculated values for $\beta=-2.0$. They agree well as a whole except for $q=0.8 \pi$ in LEB, where the experimental accuracy is rather poor due to the weak intensity. The above value of $J$ is consistent with $J=47.9 \mathrm{~K}$ obtained from the gap $\Delta$. In Fig. 31(b) we also show the data of $\alpha \neq 0$ and $\beta=-2.0$. Although HEB is concave for $\alpha=0$ irrespective of the value of $\beta$, as seen in Figs. 29(c) and (d), yet it becomes convex as $\alpha$ increases. This feature for finite $\alpha$ contradicts the experimental results. Thus, the neutron experiment supports the absence of the NNN exchange.

In Figs. 32(a) and (b) shown are the intensity $[S(q, \omega)]$

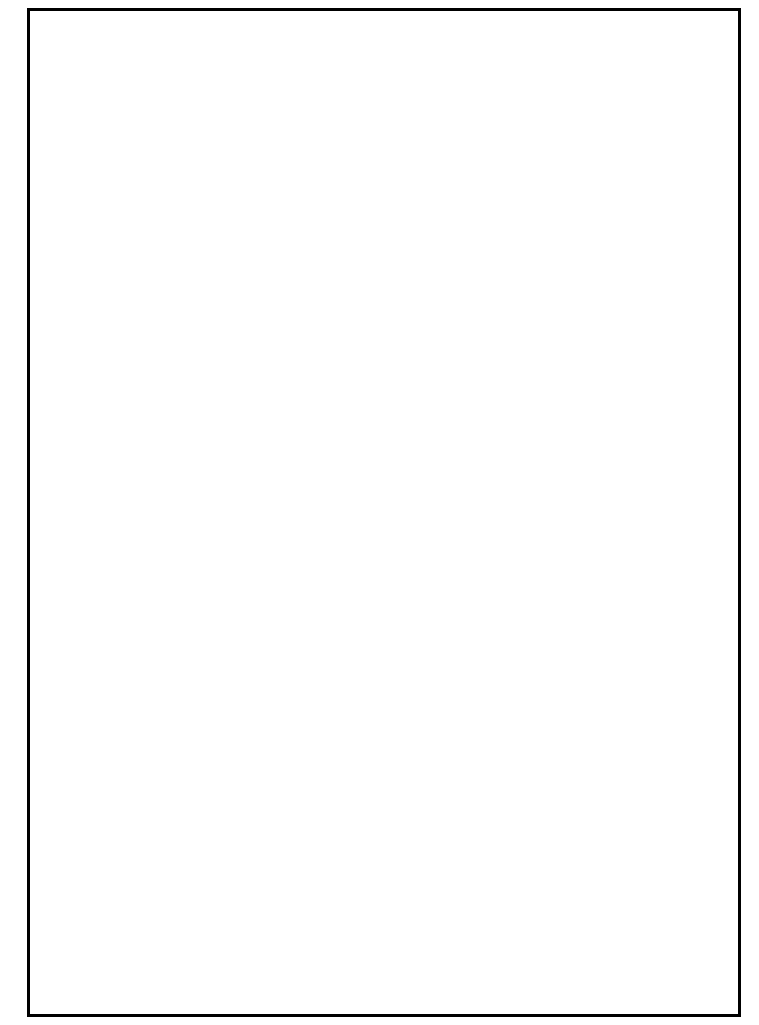

Fig. 31. Excitation energies (a) of LEB for some values of $\beta$, and (b) of HEB for a few values of $\beta(\alpha=0)$ and for some values of $\alpha(\beta=-2.0)$, as a function of $q$. The data for $L=14-26$ are simultaneously plotted, which are obtained by ED and recursion methods. A large gray circle with a error bar represents the experimental data of $10 \%$-doped $\mathrm{CuNb}_{2} \mathrm{O}_{6}{ }^{15)}$ with $J=46.9 \mathrm{~K}$.

of LEB and of HEB, respectively. In these figures we scale the experimental results so that LEB may be best fitted. For LEB in Fig. 32(a) the experimental intensity is roughly reproduced by the calculated values for $\beta=-2.0$. Kodama et al. pointed out the conspicuous intensity at $q=0.6 \pi .^{15}$ ) This is probably because the system $(\beta=-2.0)$ is in the regime of IC correlation in $S(q)$ as discussed in $\S 4.2$. Then, $S(q)$ has a peak at $q=0.515 \pi$ (See Fig.11). On the other hand, the experimental intensity for HEB [Figs. 32(b)] is considerably smaller than what the theory expects. This discordance is still an open question. 


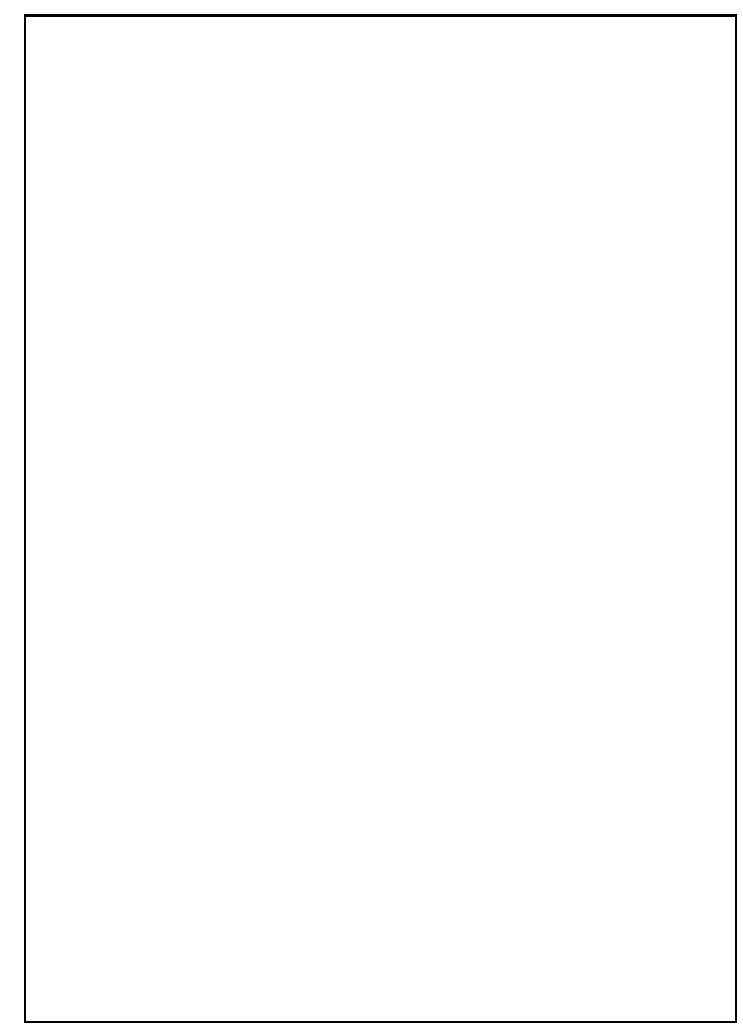

Fig. 32. Intensity $[S(q, \omega)]$ of (a) LEB for several values of $\beta$, and of (b) HEB for a few values of $\beta$; both for $\alpha=0$ and as a function of $q$. The data for $L=14-26$ are simultaneously plotted, obtained from the ED and recursion methods. The diffuse data points for $\beta=-1.5$ in (b) is owing to the overlap with the continuum spectrum around HEB. A large gray circle with a error bar represents the experimental value of $10 \%$-doped $\mathrm{CuNb}_{2} \mathrm{O}_{6}$ scaled so as to best fit the lowest-energy branch of $\beta=-2.0$.

1) Y. Ajiro et al. : Phys. Rev. B 51 (1995) 9399.

2) K. Kojima et al. : Hyperfine Interact. 104 (1997) 37.

3) M. Hase et al. : Phys. Rev. Lett. 71 (1993) 4059; B. Grenier et al. : Phys. Rev. B 57 (1998) 3444.

4) K. Manabe et al. : preprint.

5) M. Azuma et al. : Phys. Rev. B 55 (1997) R8658.

6) N. Fujiwara et al. : Phys. Rev. Lett. 80 (1998) 604.

7) M. Ito et al. : J. Phys. Soc. Jpn. 65 (1995) 2610; T. Kawae et al. : ibid. 66 (1996) 1892.

8) T. Nishikawa et al. : J. Phys. Soc. Jpn. 67 (1998) 1988.

9) I. Affleck: Phys. Rev. Lett. 62 (1989) 474; T. Sakai and M. Takahashi: Phys. Rev. B 42 (1990) 4537.

10) M. Troyer, M.E. Zhitomirsky and K. Ueda: Phys. Rev. B 55 (1997) R6117.

11) W. Geertsma and D. Khomskii: Phys. Rev. B 54 (1996) 3011; D. Khomskii et al. : Czech. J. Phys. 46 (1996) Suppl. S6, 3239.

12) J. Riera and A. Dobry: Phys. Rev. B 51 (1995) 16098; G. Castilla, S. Chakravarty and V.J. Emery: Phys. Rev. Lett. 75 (1995) 1823.

13) H. Yokoyama and Y. Saiga: J. Phys. Soc. Jpn. 66 (1997) 3617.

14) K. Fabricius et al. : Phys. Rev. B 57 (1998) 1102; A. Klümper, R. Raupach and F. Schönfeld: cond-mat/9809224.

15) K. Kodama et al. : preprint.

16) M. Nishi, O. Fujita and J. Akimitsu: Phys. Rev. B 50 (1994) 6508.

17) S. Brehmer, H.-J. Mikeska and U. Neugebauer: J. Phys. Cond. Mat. 8 (1996) 7161.
18) S. Brehmer et al. : J. Phys. Cond. Mat. 10 (1998) 1103.

19) T. Nakamura et al. : J. Phys. Cond. Mat. 9 (1997) 6401.

20) T. Tonegawa and I. Harada: J. Phys. Soc. Jpn. 56 (1987) 2153.

21) R. Bursill et al. : J. Phys. Cond. Mat. 7 (1995) 8605.

22) S.R. White and I. Affleck: Phys. Rev. B 54 (1996) 9862.

23) R. Chitra et al. : Phys. Rev. B 52 (1995) 6581.

24) B.S. Shastry and B. Sutherland: Phys. Rev. Lett. 47 (1981) 964

25) C.K. Majumdar and D.K. Ghosh: J. Math. Phys. 10 (1969) 1388, ibid. 1399; C.K. Majumdar: J. Phys. C 3 (1970) 911.

26) K. Hida: Phys. Rev. B 45 (1992) 2207, J. Phys. Soc. Jpn. 63 (1994) 2514

27) T. Kennedy and H. Tasaki: Phys. Rev. B 45 (1992) 304, Commun. Math. Phys. 147 (1992) 431.

28) M. Kohmoto and H. Tasaki: Phys. Rev. B 46 (1992) 3486.

29) S. Takada: J. Phys. Soc. Jpn. 61 (1992) 428.

30) K. Hida and S. Takada: J. Phys. Soc. Jpn. 61 (1992) 1879.

31) S. R. White: Phys. Rev. B 53 (1996) 52.

32) I. Affleck et al. : Phys. Rev. Lett. 59 (1987) 799, Commun. Math. Phys. 115 (1988) 477.

33) T. Kennedy: J. Phys. Cond. Mat. 2 (1990) 5737.

34) M. den Nijs and R. Rommelse: Phys. Rev. B 40 (1989) 4709; H. Tasaki: Phys. Rev. Lett. 66 (1991) 798.

35) K. Okamoto and K. Nomura: Phys. Lett. A 169 (1992) 433; S. Eggert: Phys. Rev. B 54 (1996) 9612.

36) F.D.M. Haldane: Phys. Rev. Lett. 60 (1988) 635; B.S. Shastry: Phys. Rev. Lett. 60 (1988) 635.

37) E.H. Lieb, T.D. Schultz and D.C. Mattis: Ann. Phys. (New York) 16 (1961) 407.

38) Since we discuss also the weight of $S(q, \omega)$, the original Brillouin zone, namely one for $S=1 / 2$ Heisenberg model, is used throughout this paper.

39) S.R. White: Phys. Rev. Lett. 69 (1992) 2863, Phys. Rev. B 48 (1993) 10345.

40) S.R. White and D.A. Huse: Phys. Rev. B 48 (1993) 3844.

41) When the $\delta$ dependence of the spin gap in eq. (2.3) is derived from the $1-\beta$ dependence in eq.(1.1), one should pay attention to the simultaneous change of $\alpha$ to $\tilde{\alpha}$. See Appendix A.

42) J.C. Bonner and H.W.J. Blöte: Phys. Rev. B 25 (1982) 6959; Z.G. Soos et al. : Phys. Rev. B 32 (1985) 3124

43) Indeed, there is an oscillation with a characteristic period in $S_{i j}$. We take up this point in $\S 4$.

44) K. Nomura: Phys. Rev. B 40 (1989) 2421.

45) I. Affleck et al. : J. Phys. A 22 (1989) 511; R.R. Singh, M.E. Fisher and R. Shanker: Phys. Rev. B 39 (1989) 2562.

46) U. Schollwöck, Th. Jolicoeur and T. Garel: Phys. Rev. B 53 (1996) 3304.

47) For instance, J. Stephenson: Phys. Rev. B 1 (1970) 4405, Can. J. Phys. 48 (1970) 1724; T. Garel and J.M. Maillard: J. Phys. C 19 (1986) L505.

48) A. Kolezhuk, R. Roth and U. Schollwöck: Phys. Rev. Lett. 77 (1996) 5142, Phys. Rev. B 55 (1997) 8928.

49) R.J. Bursill, T. Xiang and G.A. Gehring: J. Phys. A 28 (1995) 2109.

50) M. Karbach et al. : Phys. Rev. B 55 (1997) 12510.

51) S.K. Pati, R.P. Singh and D.I. Khomskii: preprint (condmat/9807270).

52) A part of the following aspects as to the local moment have been already reported in recent literature. See refs. 53-55.

53) E. Sørensen et al: preprint (cond-mat/9805386).

54) M. Laukamp et al. : Phys. Rev. B 57 (1998) 10755.

55) P. Hansen et al. : preprint (cond-mat/9805325); D. Augier et al. : preprint (cond-mat/9807265).

56) For instance, four humps appear in the $S^{z}=-2$ subspace, as we expect four spinons, there.

57) L.D. Faddeev and L.A. Takhtajan: Phys. Lett. 85A (1981) 375; F.D.M. Haldane: Phys. Rev. Lett. 50 (1983) 1153.

58) A.H. Bougourzi et al. : Phys. Rev. B 54 (1996) R12669; A.H. Bougourzi: Mod. Phys. Lett. B 10 (1996) 1237.

59) F.D.M. Haldane: Phys. Rev. B 25 (1982) 4925; D.G. Shelton, A.A. Nersesyan and A.M. Tsvelik: Phys. Rev. B 53 (1996) 8521; I. Affleck: preprint (cond-mat/9705127). 
60) G.S. Uhrig and H.J. Schulz: Phys. Rev. B 54 (1996) R9624.

61) D. Poilblanc et al. : Phys. Rev. B 55 (1997) R11941.

62) G. Bouzerar, A.P. Kampf and F. Schönfeld: preprint (condmat/9701176); G. Bouzerar, A.P. Kampf and G.I. Japaridze: Phys. Rev. B 58 (1998) 3117.

63) K. Kubo: Phys. Rev. B 48 (1993) 10552; T. Nakamura and K. Kubo: ibid. 53 (1996) 6393; D. Sen et al. : ibid. 53 (1996) 6401; T. Nakamura and S. Takada: ibid. 55 (1997) 14413.

64) One of the present authors concluded in a previous paper ${ }^{13)}$ that the value of $\tilde{\alpha}$ was $0.4-0.45$ by comparing $S(q, \omega)$ with the inelastic neutron scattering spectra for $\mathrm{CuGeO}_{3}$ in ref. 65 . Afterward, however, more elaborate examination of neutron spectra by Fujita and Arai has corrected the value to 0.3-0.4, which is compatible with that obtained by spin susceptibility, $\tilde{\alpha} \sim 0.35 .^{12,14)}$ See ref. 66 for details.

$65)$ M. Arai et al. : Phys. Rev. Lett. 77 (1996) 3649.

66) M. Fujita: Thesis (Kobe University, 1998, in Japanese).

67) M. Aïn et al. : Phys. Rev. Lett. 78 (1997) 1560.

68) S. Miyashita and S. Yamamoto: Phys. Rev. B 48 (1993) 913.

69) K.M. Kojima et al. : Phys. Rev. Lett. 79 (1997) 503.

70) H. Fukuyama, T. Tanimoto and M. Saito: J. Phys. Soc. Jpn. 65 (1996) 1182.

71) M.C. Cross and D.S. Fisher: Phys. Rev. B 19 (1979) 402.

72) T. Masuda et al. : Phys. Rev. Lett. 80 (1998) 4566.

73) H. Nakao et al. : submitted to Phys. Rev. B.

74) K. Hirota et al. : Phys. Rev. Lett. 73 (1994) 736.

75) M.C. Martin et al. : Phys. Rev. B 56 (1997) 3173.

76) This scenario is suggested by K. Hirota.

77) S. Eggert and I. Affleck: Phys. Rev. Lett. 31 (1995) 934.

78) M. Takigawa et al. : Phys. Rev. B 55 (1997) 14129.

79) M. Hagiwara et al. : Phys. Rev. Lett. 65 (1990) 3181; M.W. Meisel: preprint (cond-mat/9809077).

80) Z. Hiroi and M. Takano: Nature 377 (1995) 41.

81) D.C. Johnston: Phys. Rev. B 54 (1996) 13009; T. Imai et al. : Phys. Rev. Lett. 81 (1998) 220; R.S. Eccleston et al. : Phys. Rev. Lett. 81 (1998) 1702.

82) S. Takada and K. Kubo: J. Phys. Soc. Jpn. 60 (1991) 4026.

83) G.B. Martins, E. Dagotto and J.A. Riera: Phys. Rev. B 54 (1996) 16032; A.K. Hassan et al. : Phys. Rev. Lett. 80 (1998) 1984.

84) J.J. Borrás-Almenar et al. : Inorg. Chem. 33 (1994) 5171.

85) M. Takahashi: Phys. Rev. B 50 (1994) 3045.

86) K. Kodama et al. : J. Phys. Soc. Jpn. 67 (1998) 57.

87) T. Fukamachi et al. : J. Phys. Soc. Jpn. 67 (1998) 2107.

88) S. Mitsuda et al. : J. Phys. Soc. Jpn. 67 (1998) 1060.

89) T. Barnes and J. Riera: Phys. Rev. B 50 (1994) 6817; A.W. Garret et al. : Phys. Rev. Lett. 79 (1997) 745.

90) T. Nakamura and K.Okamoto: preprint; H. Tanaka et al. : J. Phys. Soc. Jpn. 65 (1996) 1945.

91) The difference between $L=16$ and 12 for $\chi(T)$ and $C(T)$ is hardly observed for sufficiently dimerized cases $(1 \gg \beta \gg$ $-\infty)$.

92) J. Kanamori: J. Phys. Chem. Solids 10 (1959) 87; J. Goodenough: Magnetism and the Chemical Bond (John Wiley and Sons, New York, 1963).

93) We owe the values in Table II to M. Sato's group of Nagoya University. As $x$ increases, the accuracy of fit naturally becomes poor.

94) H. Kikuchi et al. : J. Phys. Soc. Jpn. 64 (1995) 3429. 